\title{
Research on multi-sensor measurement system and evaluation method for roundness and straightness errors of deep-hole parts
}

\section{Ci Song}

Xibin Wang

zhibing Liu ( $\square$ liuzhibing@bit.edu.cn )

Beijing Institute of Technology

Hui Chen

\section{Research Article}

Keywords:

Posted Date: February 17th, 2021

DOI: https://doi.org/10.21203/rs.3.rs-201791/v1

License: (c) (1) This work is licensed under a Creative Commons Attribution 4.0 International License. Read Full License 


\title{
Research on multi-sensor measurement system and evaluation method for roundness and straightness errors of deep-hole parts
}

\author{
Ci Song ${ }^{1}$, Xibin Wang ${ }^{1}$, Zhibing Liu $^{1 *}$ and Hui Chen ${ }^{1}$ \\ ${ }^{1}$ School of Mechanical Engineering, Beijing Institute of Technology, Beijing 100081, China \\ Corresponding author: Zhibing Liu E-mail: liuzhibing@bit.edu.cn
}

\begin{abstract}
Precision deep-hole parts are widely used in various fields of industrial production and their machining quality has a great impact on fatigue limit, geometric accuracy, and stability of products. Since roundness and straightness errors are essential technical indexes to evaluate the machining quality of deep-hole parts, accurate measurement and effective evaluation of them are of great significance to ensure the performance of related products. A multi-sensor integrated device that can measure two kinds of shape errors simultaneously was developed based on laser displacement sensor, two-dimensional position-sensitive detector, angle sensor, and laser distance sensor. Aiming at the problem of roundness error evaluation, the solution process of the control points of the minimum zone circle was optimized by calculating the distance between points and searching according to the polygon removal rule. Besides, the rotating projection method was used to evaluate the straightness error effectively. Eventually, the effectiveness of the measuring device and the shape error evaluation method was verified by experimental research.
\end{abstract}

Keywords: Deep-hole parts, roundness error, straightness error, multi-sensor integrated measuring device, shape error evaluation method

\section{Introduction}

With the rapid development of modern equipment manufacturing industry, high-precision deep-hole parts have been widely used in the fields such as aviation, shipping, metallurgy, and automobile. As precision components, the roundness and straightness errors of deep-hole parts could directly affect the overall performance of products. For example, the roundness deflection for hole parts such as engine cylinders used in strict shaft-hole fitting occasions would reduce the tightness of shaft-hole fitting and affect the efficiency of energy conversion. Furthermore, the bending of the axis would cause vibration and tangential deformation during reciprocating motion, resulting in a negative effect on the movement ability and even potential safety hazards. For non-fitting parts such as metallurgical rollers, the roundness change and axis deviation would lead to irregular shapes of cooling channels, making the surface temperature distribution uneven and affecting the cooling performance of rollers. Therefore, accurate measurement and evaluation of roundness and straightness errors of deep-hole parts is a crucial basis to ensure the accuracy of parts.

The measurement of roundness and straightness errors can be divided into contact and non-contact modes. In contact measurement, the measured parameters are obtained by contacting the probe with the measured surface, and the typical measuring equipment includes coordinate measuring machine (CMM) and roundness tester. Besides, the acoustic emission rotary probe was designed by Salah et al. [1] to measure micro hole roundness error, and the three-dimensional inner hole roundness and straightness measuring system was developed by Chang et al. [2] and composed of the capacitive probe and rotary table; they both adopted contact measurement method. Its advantage lies in the high measurement accuracy. However, this kind of device is limited by its own structure, which cannot measure the inaccessible area of probe and has strict requirements on the measurement conditions.

Due to the emergence of technologies such as photoelectric, electromagnetic, and image, non-contact measurement method has been applied more and more because of it has advantages such as effective protection of measured surface quality and is suitable for extreme space. The method adopts some 
equipment such as laser displacement sensor, capacitance sensor, and charge-coupled device (CCD) camera to obtain data information without touching the measured surface. For example, the diameter and roundness error measuring system was developed by Mekid and Vacharanukul [3] based on the corresponding relationship between displacement and light intensity; the cylindrical non-contact capacitance probe was used by Ma et al. [4]; the deep-hole inner surface parameter comprehensive measurement device was designed by Akio et al. [5], consisting of laser emitter, DC motor, measurement unit, and optical element for detecting posture; the adjustable five-dimensional measuring system was established by Ma et al. [6]; the measuring device consisting of two high-resolution single-lens reflex cameras was proposed by Zatočilová et al. [7]; additionally, the system designed by Jurevicius and Vekteris [8-10] was based on the phase difference coding of modulated optical signals emitted by two photodiodes. All of them are successful typical applications of non-contact mode in the field of shape error measurement and have been improved to varying degrees regarding measurement rationality and accuracy. However, these current applications are only for hole parts with small diameter or small ratio of length to diameter, and only one measured parameter such as roundness or straightness errors can be obtained in a measurement process. Therefore, there are still few measuring methods which are really suitable for deep-hole parts and can accurately obtain the roundness and straightness errors at the same time.

Similarly, the error evaluation of the measurement point set is the key to obtain the shape error value. There are four basic methods for evaluating roundness error: least square circle (LSC), minimum circumscribed circle (MCC), maximum inscribed circle (MIC), and minimum zone circle (MZC). Considering that the four methods have different reference circles, how to acquire the ideal reference circle is essential to ensure the evaluation accuracy. Li and Shi [11] proposed the concept of minimum zone line and used it as the control line of MZC. Meanwhile, the MCC and MIC reference circles of the measurement point set were obtained [12-13] according to the three relations of relative diameters. By establishing convex hull, Gadelmawla [14] employed the size relationship of arc radius to remove invalid points in the measurement point set in turn and circularly searched in the remaining point set until the control points of MCC, MIC, and MZC were searched out. Lei et al. [15] set a circular area near the center of LSC and obtained MCC, MIC, and MZC center points using the proposed polar coordinate transformation algorithm. Goch and Lubke [16] applied the improved Chebyshev algorithm to approximate geometric elements and acquired the maximum internal element and the minimum external element. Besides, Li and Shi [17] revealed that the control points of MCC, MIC, and MZC are interrelated, and their control points can be solved mutually.

Since the axis of deep-hole parts is a spatial straight line, its straightness error evaluation belongs to the spatial straightness error evaluation. Among many evaluation methods, the two-point connection method is the simplest while its accuracy is the worst; least square method (LSM) is simple and fast. Both of them can meet the general precision requirements. However, they are not suitable for precision machining. There is no unified method for solving the minimum area, though the error value evaluated by the minimum zone method (MZM) is the smallest and unique. For example, Dhanish et al. [18] adopted the mode of iterative calculation of measurement point set and searched for control point combinations; the minimum parallelepiped envelope method was used by Huang et al.; Cho and Kim [19] proposed a linearization algorithm based on data envelopment analysis. Moreover, spatial straightness error evaluation is a nonlinear optimization problem. To address this issue, Ding et al. [2021] combined semi-definite programming and Chebyshev approximation theory; Calvo et al. [22] applied a calculation method of coordinate vector integration. For the spatial problem, projection can play the 
role of "dimension reduction", and the problem of spatial straightness error can be transformed into the problem of solving the MCC on the plane by projecting the measurement point along the projection axis to the plane perpendicular to it. Endrias et al. [23] continuously rotated the projection axis according to the three situations (three points, four points, and five points in contact between the minimum containment zone and the measurement point set) until the control points conforming to the minimum zone was searched.

For shape error evaluation, no one method is universal. The data point sets obtained by different measuring devices have their own applicable evaluation methods [24]. Therefore, in order to obtain the accurate and effective error values, it is necessary to equip the newly developed measuring device with the suitable error evaluation method.

In this paper, the measurement and evaluation of roundness and straightness errors of deep-hole parts are deeply investigated, and a convenient roundness and straightness error measuring device is developed to measure two kinds of shape errors simultaneously. Furthermore, the improved MZC method is applied to evaluate roundness error, and the rotating projection method based on the principle of the minimum region is employed to solve the straightness error.

\section{Measuring principle}

Roundness error refers to the radius difference between the two smallest concentric circles containing the actually measured contour in the same section. As illustrated in Fig.1, $M_{c}$ represents the actually measured contour, $Z_{c}$ denotes the smallest contained concentric circle, and the radius difference $f_{c}$ indicates the roundness error. Straightness error is defined as the diameter of the smallest cylindrical surface containing the measured axis. It is extracted from the fitting center trajectories of several sections of the measured cylindrical surface. In Fig.2, $L$ denotes the measured extraction axis, $S$ represents the measured section, $Z_{s}$ refers to the smallest containment cylinder of the measured extraction axis, and its diameter $f_{s}$ is the straightness error. Therefore, the measurement of roundness error is the process of determining the actually measured contour while that of straightness error is the process of extracting the actual axis of deep-hole parts.

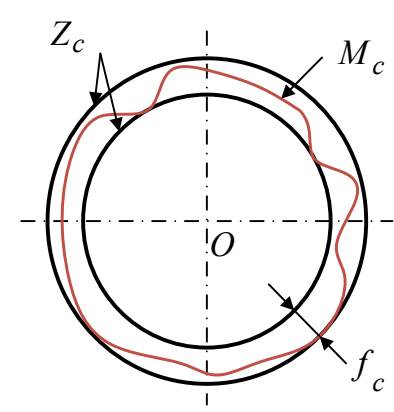

Fig.1 Schematic diagram of roundness error.

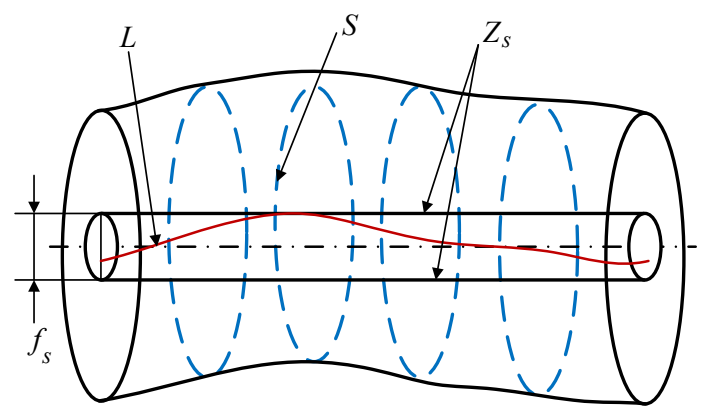

Fig.2 Schematic diagram of straightness error.

According to the measuring principle of roundness and straightness errors, a multi-sensor integrated measuring device has been developed by effectively combining laser displacement sensor, angle sensor, position-sensitive detector (PSD), and laser distance sensor. The schematic diagram of the measuring device is displayed in Fig.3.

Initially, the laser displacement sensor is driven by the driving components to scan the contour point by point in the measured section and conduct equal angle sampling. This sampling method can not only equalize the angles between adjacent sample points in the same section but also ensure the same number of sample points in each measured section, making it convenient for subsequent evaluation. Additionally, the displacement value measured by the laser displacement sensor is transformed into the rectangular 
coordinate value of the corresponding measurement point according to the geometric relationship. The schematic diagram of transformation from displacement value to coordinate value is illustrated in Fig.4, where $O$ represents the center of the measured section, $O^{\prime}$ denotes the rotation center of the sensor and the origin of the rectangular coordinate system, the horizontal direction is the $x$-axis, and $P$ indicates the measurement point. The rectangular coordinates of $P$ can be calculated by Eq. (1).

$$
\left\{\begin{array}{c}
\left(\left(r_{i}+a\right) \cos \theta_{i}-b \sin \theta_{i},\left(r_{i}+a\right) \sin \theta_{i}+b \cos \theta_{i}\right), \quad \text { while sensor rotates counterclockwise; } \\
\quad\left(\left(r_{i}+a\right) \cos \theta_{i}+b \sin \theta_{i},-\left(r_{i}+a\right) \sin \theta_{i}+b \cos \theta_{i}\right), \quad \text { while sensor rotates clockwise. }
\end{array}\right.
$$

where $r_{i}$ denotes the measured displacement value of the $i$-th measurement point, $\theta_{i}$ refers to the included angle between the laser beam and the $x$-axis, $a$ represents the calibrated distance between the laser emission hole and the rotation axis, and $b$ indicates the eccentricity between the rotation axis and the laser emission hole caused by machining or assembly error. Furthermore, the $\mathrm{z}$ coordinate value of point can be obtained by adding the moving distance $c$ of the device along the axial direction measured by the laser distance sensor and the horizontal distance $l$ between the center of PSD photosensitive surface and the laser emission hole of the laser displacement sensor.

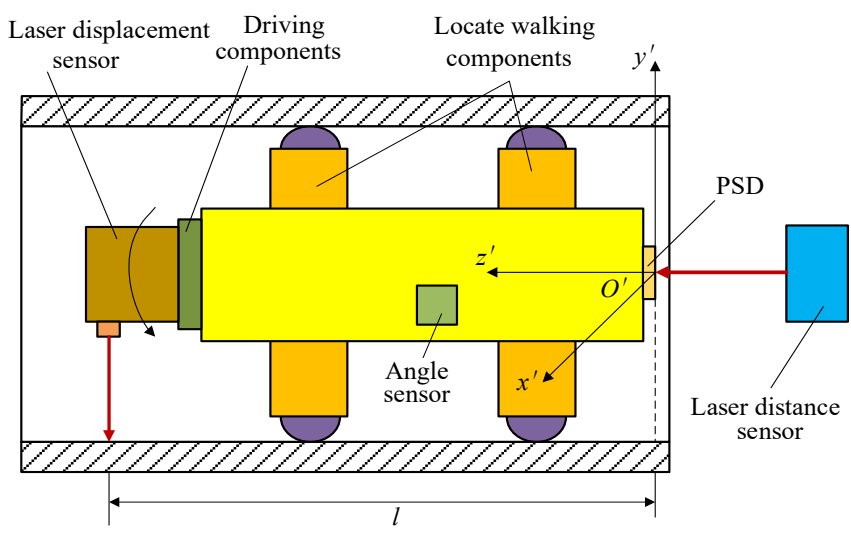

Fig.3 Schematic diagram of the measuring device.

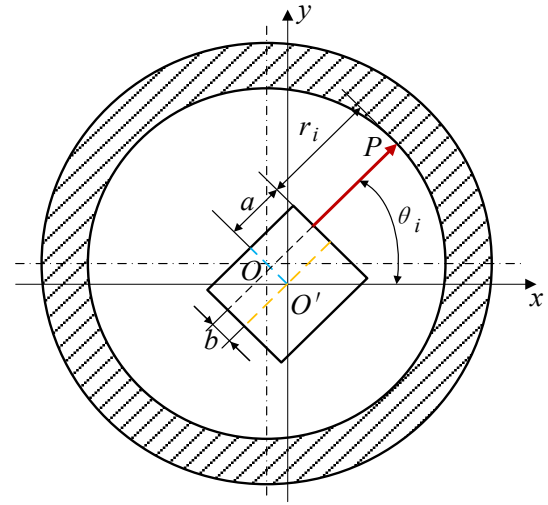

Fig.4 Schematic diagram of transformation from displacement value to coordinate value.

Since PSD is fixed on the measuring device, the center of its photosensitive surface is located on the axis of the device. Then, the laser distance sensor after leveling and straightening is placed on one side and its emitted laser is taken as the reference axis of the inner hole. In the measured section, PSD and laser distance sensor are combined to record the displacement offset of the device, and the angle sensor reflects the attitude variation. Thus, the coordinate values of the point set in the measurement coordinate system are transformed into those in the absolute coordinate system. As illustrated in Fig.5, the measurement coordinate system established with the center of PSD as the origin is $o^{\prime}-x^{\prime} y^{\prime} z^{\prime}$, the absolute coordinate system is $o-x y z$, and $o^{\prime}-x^{\prime} y^{\prime} z^{\prime}$ coincides with $o-x y z$ in the initial state. The angle sensor obtains the Euler angles, and the measurement coordinate system $o^{\prime}-x^{\prime} y^{\prime} z^{\prime}$ rotates around its own $z$-axis, $y$-axis, and $x$-axis by angles $\varphi, \theta$, and $\psi$, respectively, to reach the final posture.

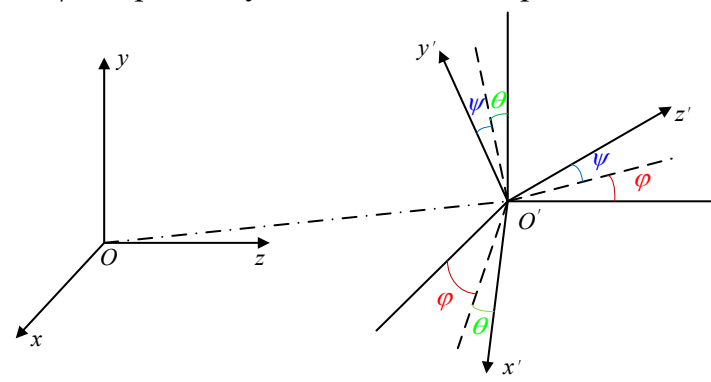

Fig. 5 Transformation from measurement coordinate system to absolute coordinate system. 
The coordinates of measurement points in the $o^{\prime}-x^{\prime} y^{\prime} z^{\prime}$ obtained by the laser displacement sensor are $\left(x^{\prime}, y^{\prime}, z^{\prime}\right)$ while those in the $o-x y z$ are $(x, y, z)$. According to the posture transformation relation of $o^{\prime}-x^{\prime} y^{\prime} z^{\prime}$, $(x, y, z)$ can be calculated by Eq. (2).

$$
\left[\begin{array}{l}
x \\
y \\
z \\
1
\end{array}\right]=\left[\begin{array}{cccc}
\cos \theta \cos \phi & \sin \psi \sin \theta \cos \phi-\cos \psi \sin \phi & \cos \psi \sin \theta \cos \phi+\sin \psi \sin \phi & a \\
\cos \theta \sin \phi & \sin \psi \sin \theta \sin \phi+\cos \psi \cos \phi & \cos \psi \sin \theta \sin \phi-\sin \psi \cos \phi & b \\
-\sin \theta & \sin \psi \cos \theta & \cos \psi \cos \theta & 0 \\
0 & 0 & 0 & 1
\end{array}\right]\left[\begin{array}{l}
x^{\prime} \\
y^{\prime} \\
z^{\prime} \\
1
\end{array}\right]
$$

where $a$ and $b$ represent the horizontal and vertical displacement offsets recorded by PSD, respectively.

After the coordinate values of the point set in the absolute coordinate system are obtained, the least square circle method is used to solve the center of the measured section. Depending on locate walking components, multiple measured sections can be obtained by moving the device back and forth. Roundness error can be calculated by a relevant evaluation algorithm in each measured section. Then, the center of each measured section is connected in turn to obtain the axis of deep-hole parts and realize the solution of straightness error.

\section{Evaluation method}

\subsection{Roundness Error-Improved MZC Method}

The MZC method is to evaluate the roundness error using the MZC center of the point set, and the MZC refers to two concentric circles that meet the following conditions: a) all the measurement points are on or between the two concentric circles; b) the radius difference of them should be the smallest. The determining criterion: there are at least four inner and outer points in contact with concentric circles, and the two-point connecting line on the outer circle keeps intersecting with the two-point connecting line on the inner circle, called intersection criterion.

According to the relationship among MCC, MIC, and MZC [17], the control points of MZC can be solved by the control points of MCC and MIC. The construction process of MCC and MIC follows the principle of acute triangle or diameter: three points on the solution circle are in contact with the measurement point set, forming an acute triangle; or only two measurement points are located on the solution circle, and the midpoint of the two points is the center. Therefore, the two outer control points of MZC can be selected from the control points of MCC while the control points of MIC can be chosen as the two inner control points of MZC.

Generally, solving the MCC and MIC needs to construct the outer convex hull point set and the inner convex hull point set respectively [25], so as to simplify the calculation and obtain the measurement points that meet the requirements. However, the construction process is extremely complicated, and the non-uniqueness of the inner convex hull point set would cause great uncertainty to the evaluation results. Therefore, based on the distance between measurement points and the polygon removal rule, the search process of control points is optimized, and the control points of MCC and MIC are determined quickly and accurately without solving convex hull point set. The specific evaluation process of the improved MZC method for evaluating roundness error is described as follows:

(1) The MCC control points.

For a given measurement point set $P_{i}\left(x_{i}, y_{i}\right), i=1,2, \cdots, n$, the center point $O_{\text {och }}$ of the point set is calculated by Eq. (3).

$$
x_{\mathrm{och}}=\frac{1}{m} \sum_{i=1}^{m} x_{j}, y_{\mathrm{och}}=\frac{1}{m} \sum_{i=1}^{m} y_{j}
$$

The distance between each point in the point set and $O_{\text {och }}$ is successively calculated to obtain the point $P_{1}$ farthest from $O_{\text {och }}$ and the straight line $P_{1} O_{\text {och. }}$. Then, we search the points $P_{2}$ and $P_{3}$ on both sides of 
the line $P_{1} O_{\text {och }}$ with the largest distance from it, as presented in Fig.6(a). The points $P_{2}, P_{3}$, and $P_{1}$ together form a triangle. For three points $P_{i-1}\left(x_{i-1}, y_{i-1}\right), P_{i}\left(x_{i}, y_{i}\right)$ and $P_{i+1}\left(x_{i+1}, y_{i+1}\right)$, the angle $\theta$ corresponding to $P_{i}$ could obtained by Eq. (4). And whether the formed triangle is obtuse triangle could be judge.

$$
\theta=\arccos \frac{\left(x_{i-1}-x_{i}\right)\left(x_{i+1}-x_{i}\right)+\left(y_{i-1}-y_{i}\right)\left(y_{i+1}-y_{i}\right)}{\sqrt{\left(x_{i-1}-x_{i}\right)^{2}+\left(y_{i-1}-y_{i}\right)^{2}} \sqrt{\left(x_{i+1}-x_{i}\right)^{2}+\left(y_{i+1}-y_{i}\right)^{2}}}
$$

When it belongs to the obtuse triangle, the longest side is taken as the diameter, and the midpoint of it is taken as the center of circumscribe circle. If it contains all the measurement points, the circle is the MCC we expected. Assuming that some measurement points are located on or outside the circle, the two points farthest from the longest side on both sides of it are solved, forming a quadrilateral with two endpoints of the longest side. For each point of the quadrilateral, the radius of the circle passing through it and its adjacent points is calculated. After the point with the maximum radius is removed, the remain points constitute a new triangle.

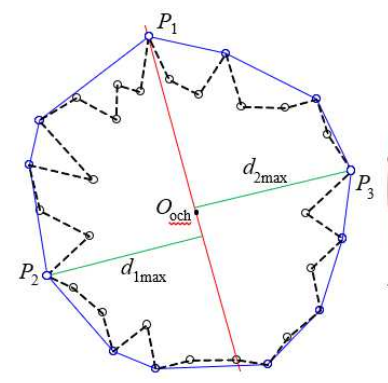

(a)

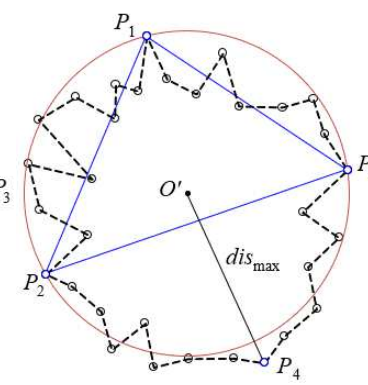

(b)

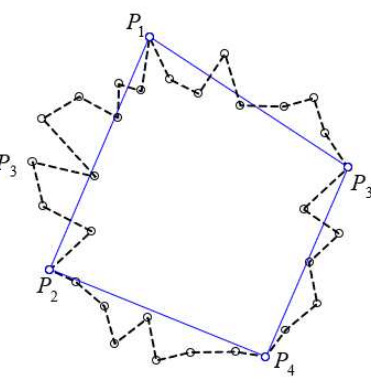

(c)

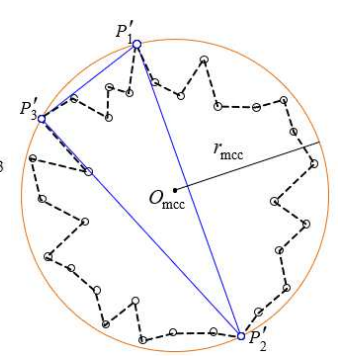

(d)

Fig.6 (a) Search the points $P_{2}$ and $P_{3}$ on both sides of the line $P_{1} O_{\text {och }}$ with the largest distance from it; (b) Search the point $P_{4}$ with the largest distance from the center $O^{\prime}$ of MCC; (c) The point $P_{4}$ is added into the triangle to form a quadrilateral; (d) The control points of MCC.

On the contrary, in the acute triangle, the center $O^{\prime}\left(c_{x}, c_{y}\right)$ and radius $R_{r}$ of the circumscribe circle passing through three points are calculated according to Eqs. (5) (6). If it contains all the measurement points, the circle is the MCC we expected. In a certain calculation process, the triangle $P_{1} P_{2} P_{3}$ is acute and there are some measurement points outside the circumscribe circle (Fig.6(b)); then, the point $P_{4}$ with the largest distance from the center is searched. Fig.6(c) illustrates that $P_{4}$ and the triangle $P_{1} P_{2} P_{3}$ constitute a quadrilateral $P_{1} P_{2} P_{4} P_{3}$. In the quadrilateral, the radius of arc determined by three adjacent points is calculated successively, and the middle point corresponding to the maximum radius value is removed to form a new triangle.

$$
c_{x}=-\frac{B}{2 A} 、 c_{y}=-\frac{C}{2 A} 、 R_{r}=\sqrt{\left(x_{1}-c_{x}\right)^{2}+\left(y_{2}-c_{y}\right)^{2}}
$$

where,

$$
\left\{\begin{array}{l}
A=x_{1}\left(y_{2}-y_{3}\right)-y_{1}\left(x_{2}-x_{3}\right)+x_{2} y_{3}-x_{3} y_{2} \\
B=\left(x_{1}^{2}+y_{1}^{2}\right)\left(y_{3}-y_{2}\right)+\left(x_{2}^{2}+y_{2}^{2}\right)\left(y_{1}-y_{3}\right)+\left(x_{3}^{2}+y_{3}^{2}\right)\left(y_{2}-y_{1}\right) \\
C=\left(x_{1}^{2}+y_{1}^{2}\right)\left(x_{2}-x_{3}\right)+\left(x_{2}^{2}+y_{2}^{2}\right)\left(x_{3}-x_{1}\right)+\left(x_{3}^{2}+y_{3}^{2}\right)\left(x_{1}-x_{2}\right)
\end{array}\right.
$$

The above steps are repeated until the circumscribe circle can contain all the measurement points. Then, the circumscribe circle is the MCC. In Fig.6(d), the triangle vertices corresponding to the circle represent the control points of MCC.

(2) The MIC control points.

For the same measurement point set $P_{i}\left(x_{i}, y_{i}\right), i=1,2, \cdots, n$, the center point $O_{\mathrm{p}}$ of the point set is 
calculated by Eq. (3). Then, the distance between each point in the point set and $O_{\mathrm{p}}$ are solved in turn to obtain the point $P_{1}$ with the shortest distance from $O_{\mathrm{p}}$ and the straight line $P_{1} O_{\mathrm{p}}$. As presented in Fig.7(a), the points $P_{2}$ and $P_{3}$ with the shortest distance from the line $P_{1} O_{\mathrm{p}}$ on both sides are searched, and they form a triangle together with the point $P_{1}$. Besides, whether the triangle is obtuse is judged according to Eq. (4).

When it belongs to the obtuse triangle, the longest side is taken as the diameter, and the midpoint of it is taken as the center of circumscribe circle. If all the measurement points are on or outside the circumscribe circle, the circle is the MIC we expected. In a certain calculation process, the triangle $P_{1} P_{2} P_{4}$ is an obtuse triangle and some points are inside the circumscribe circle (Fig.7(b)); then, the two points $P_{1}$ and $P_{5}$ with the smallest distance from the longest side $P_{2} P_{4}$ on both sides are solved. Besides, the points $P_{1} 、 P_{5} 、 P_{2}$ and $P_{4}$ constitute a quadrilateral $P_{1} P_{2} P_{5} P_{4}$, as exhibited in Fig.7(c). In the quadrilateral, the radius of arc determined by adjacent three points is calculated in turn, and the middle point corresponding to the minimum radius value is removed to form a new triangle.

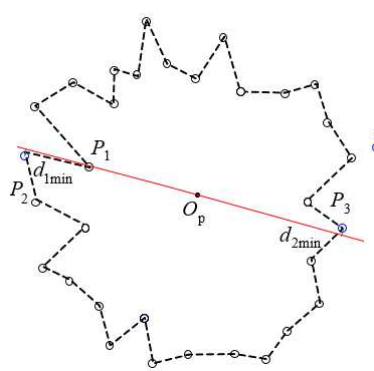

(a)

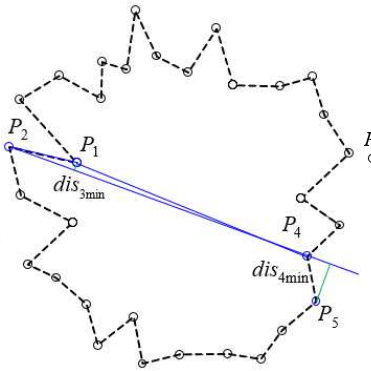

(b)

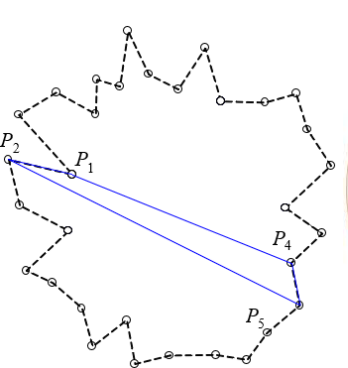

(c)

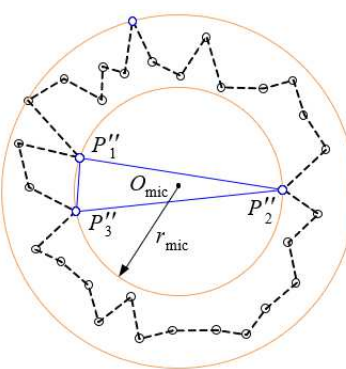

(d)

Fig.7 (a) Search that points $P_{2}$ and $P_{3}$ on both side of $P_{1} O_{\mathrm{p}}$ with the shortest distance from $P_{1} O_{\mathrm{p}}$; (b) Search the points $P_{1}$ and $P_{5}$ on both sides of the longest side $P_{2} P_{4}$ with the shortest distance from it; (c) The points $P_{1}$ and $P_{5}$ form a quadrilateral with $P_{2}$ and $P_{4}$; (d) The control point of the MIC.

On the contrary, in the acute triangle, the center and radius of the circumscribe circle passing through three points are calculated according to Eqs. (5) (6). If all the measurement points are on or outside the circumscribe circle, the circle is the MIC we expected. Assuming that some measurement points are inside the circle, the point with the smallest distance from the center of the circle is solved, and a quadrilateral is formed with the three vertices of the triangle. For each point of the quadrilateral, the radius of the circle passing through it and its adjacent points are calculated. After the point with the minimum radius is removed, the remain points constitute a new triangle.

The above steps are repeated until all the points are on or outside the circumscribe circle. Then, the circle is the MIC. In Fig.7(d), the triangle vertices corresponding to the circle represent the control points of MIC.

(3) The MZC control points.

In Fig.8(a), for the same measurement point set $P_{i}\left(x_{i}, y_{i}\right), i=1,2, \cdots, n$, the MCC control points are stored into the external control point set $C_{\max }$ while the MIC control points are stored into the internal control point set $C_{\min }$. According to the criterion of judging the MCC and MIC, the number of control points has three combinations of "3-3", "3-2", or "2-3". Therefore, the total number of control points $v$ in $C_{\max }$ and $C_{\min }$ may be 5 or 6 , respectively. Besides, two points from $C_{\max }$ and $C_{\min }$ are selected, respectively, to build a concentric circle with $3^{v-4}$ cases in total.

Every time, the points selected from $C_{\max }$ are $P_{1}\left(x_{1}, y_{1}\right)$ and $P_{2}\left(x_{2}, y_{2}\right)$, and the points selected from $C_{\min }$ are $P_{3}\left(x_{3}, y_{3}\right)$ and $P_{4}\left(x_{4}, y_{4}\right)$. The center of the concentric circle $\left(x_{x}, y_{y}\right)$ is calculated by Eq. (7). 


$$
\left\{\begin{array}{c}
x_{x}=\frac{\left(k_{1} x_{2 \text { mid }}-k_{2} x_{1 \text { mid }}\right)+k_{1} k_{2}\left(y_{1 \mathrm{mid}}-y_{2 \mathrm{mid}}\right)}{k_{1}-k_{2}}, y_{y}=y_{1 \text { mid }}+\frac{1}{k_{1}-k_{2}}\left(x_{2 \mathrm{mid}}-x_{1 \mathrm{mid}}\right), \text { while } k_{1} \neq k_{2} \neq 0 \\
x_{x}=x_{1 \mathrm{mid}}, y_{y}=y_{2 \mathrm{mid}}+\frac{1}{k_{2}}\left(x_{x}-x_{2 \mathrm{mid}}\right), \text { while } k_{1}=0 \\
x_{x}=x_{2 \mathrm{mid}}, y_{y}=y_{1 \mathrm{mid}}+\frac{1}{k_{1}}\left(x_{x}-x_{1 \mathrm{mid}}\right), \text { while } k_{2}=0
\end{array}\right.
$$

where $k_{1}$ denotes the slope of $P_{1} P_{2}, k_{2}$ denotes the slope of $P_{3} P_{4}, x_{1 \text { mid }}$ and $y_{1 \text { mid }}$ denote the abscissa and ordinate of the midpoint of $P_{1} P_{2}$, respectively, and $x_{2 \text { mid }}$ and $y_{2 \text { mid }}$ denote the abscissa and ordinate of the midpoint of $P_{3} P_{4}$, respectively.

For all the $3^{v-4}$ cases, whether the concentric circle is constructed in each case is calculated with all the points in $C_{\max }$ and $C_{\min }$. As indicated in Fig.8(b), $P_{1}, P_{2}, P_{3}$, and $P_{4}$ corresponding to the circle with the smallest radius difference are selected for subsequent calculation when the concentric circle can contain all the points in $C_{\max }$ and $C_{\min } . P_{1}$ and $P_{2}$ are stored into the point set $D_{\max }$ while $P_{3}$ and $P_{4}$ are stored into $D_{\text {min. }}$. Then, the radius $r_{\text {out }}$ and $r_{\text {in }}$ of the concentric circle and the maximum distance $d_{\max }$ and minimum distance $d_{\min }$ from each point in the measurement point set to the center of the circle are calculated. In Fig.8(c), if $d_{\max }>r_{\text {out}}$, the point corresponding to $d_{\max }$ is added into $D_{\max }$; if $d_{\min }<r_{\text {in }}$, the point corresponding to $d_{\operatorname{mim}}$ is added into $D_{\min }$. Furthermore, $D_{\max }$ and $D_{\min }$ are assigned to $C_{\max }$ and $C_{\min }$, respectively, and the search process is repeated until all the distances from measurement points to the center of the circle meet $r_{\text {out }}>d_{i}>r_{\text {in. }}$. The selected four points are the control points of MZC, as presented in Fig.8(d).

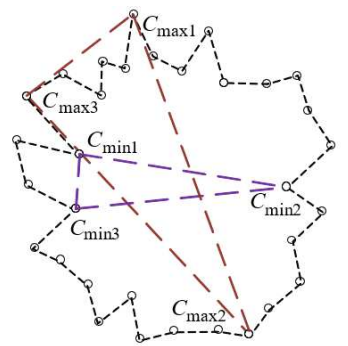

(a)

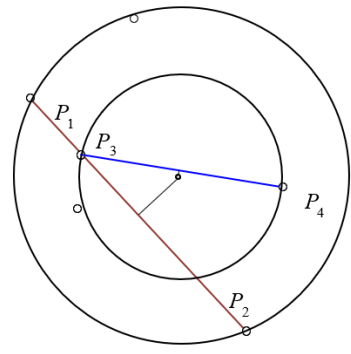

(b)

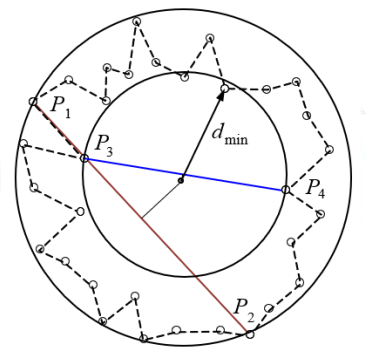

(c)

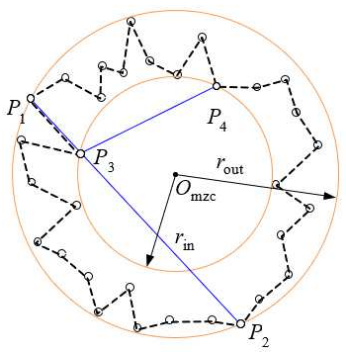

(d)

Fig.8 (a) The internal and external control points; (b) The concentric circle constructed contains internal and external control points; (c) The point corresponding to $d_{\max }$ is added into $D_{\max }$; (d) Roundness error evaluation

(4) Roundness error evaluation

Ultimately, roundness error is evaluated by Eq. (8).

$$
f_{\text {mzc }}=r_{\text {out }}-r_{\text {in }}
$$

\subsection{Straightness Error-Rotating Projection Method}

For straightness error evaluation, the projection method can transfer the solution process from threedimensional space to two-dimensional plane, reducing the amount of calculation and the difficulty of solution. On the two-dimensional plane, the minimum zone containing all measurement points is analyzed to evaluate the straightness error, and the points located on the boundary of the minimum zone are called the control points.

The traditional projection method projects the measurement points to the vertical plane perpendicular to the least square midline, transforming the spatial straightness problem into the solution of the plane MCC. There are significant disadvantages in this process. For example, only by projecting to multiple planes, the distribution of measurement points can be fully reflected, and projection suppression points 
would be produced, leading to the omission of control points. Therefore, the rotating projection method proposed by Chen Hui et al. [26] based on the principle of the minimum zone is applied to the straightness error evaluation, verifying that this method has good solution speed and operation accuracy.

The evaluation process of the rotating projection method is detailed as follows. Firstly, by rotating the coordinate system, the least square midline of measurement point set passes through the origin of evaluation coordinate system $o^{\prime}-x^{\prime} y^{\prime} z^{\prime}$ and coincides with the $z^{\prime}$-axis. Then, the measurement points are rotating projected onto the $y^{\prime} o^{\prime} z^{\prime}$ plane around the least-squares midline, and the distance relationship between each point and the midline is preserved and transformed into the $y^{\prime} o^{\prime} z^{\prime}$ plane. Consequently, the spatial straightness error evaluation problem is transformed into the plane straightness evaluation problem. Afterward, three measurement points in the $y^{\prime} o^{\prime} z^{\prime}$ plane are selected as initial control points, and the control line determined by them is solved according to the equidistant vertical deviation between the control points and the control line. The maximum distance $d_{\max }$ between measurement points and the control line is searched. When the distance is greater than the distance $d_{\mathrm{c}}$ between control point and control line, the corresponding measurement point is added to the control point set, and the redundant points are removed according to the distance proportional coefficient to form a new control point set. This process is repeated until $d_{\max }=d_{\mathrm{c}}$. Moreover, the zone determined by the control line is the smallest zone containing the plane measurement points, and the axis straightness error is $f=2 d_{\max }$.

\section{Measurement and evaluation system}

Based on the measuring principle of roundness and straightness errors, the mechanical structure of the measuring device, measuring units such as sensors and driving components such as servo motors, were explored, and a new measuring device was developed.

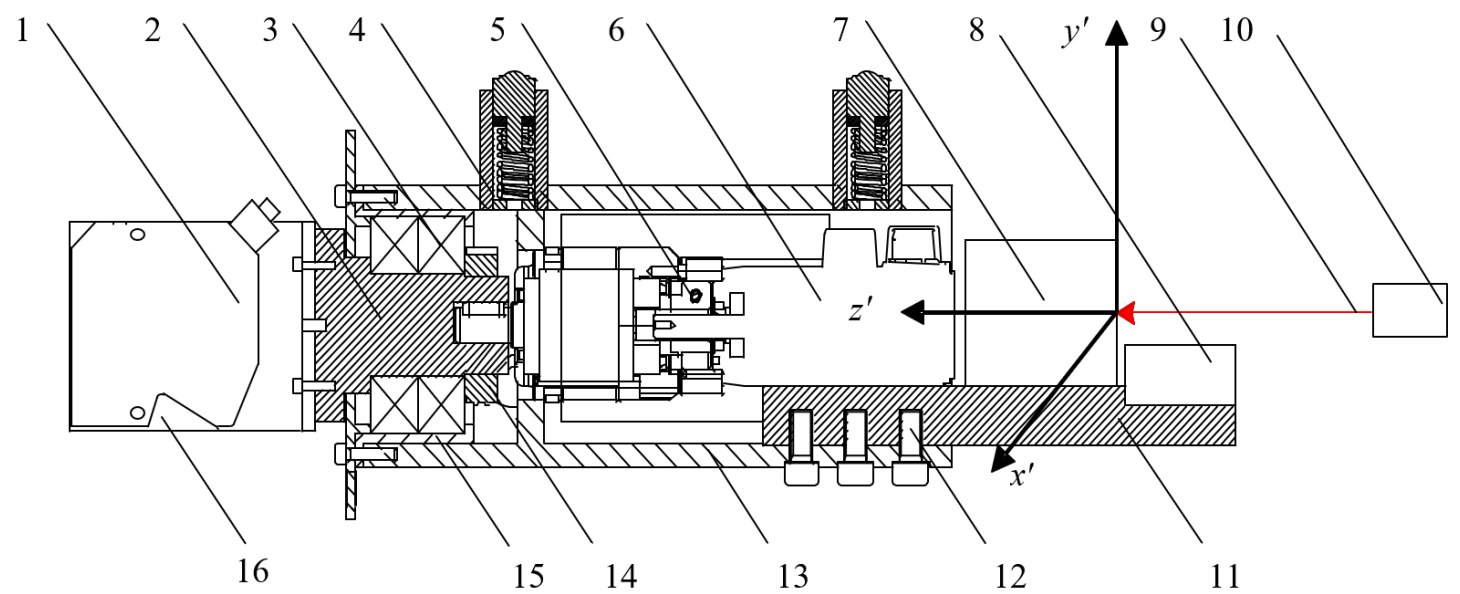

1-Laser displacement sensor, 2- Rotating shaft, 3-Angular contact ball bearing set, 4-Supporting arm, 5-Reducer,

6-Servo motor, 7-Position sensitive detector, 8-Angle sensor, 9-Laser, 10-Laser distance sensor, 11-Motor

supporting block, 12-Screw, 13-Outer cylinder, 14-Bearing lock nut, 15-Sleeve, 16-Mounting bracket

Fig.9 Overall structure diagram of measuring device.

The measuring device is mainly composed of measuring unit, driving unit, and locate walking unit. Its overall structure is displayed in Fig.9. The measuring unit consists of LK-H050 laser displacement sensor from KEYENCE, 6-axis WT901C485 angle sensor, PSD 2D-9 position-sensitive detector from TEM Messtechnik $\mathrm{GmbH}$, and industrial laser distance sensor. Besides, the driving unit includes ECM-A3HCY0401 servo motor from Delta and PA050-C-0108-A0825 reducer. The locate walking unit is composed of three supporting arms with a circumferential interval of 120 degrees to ensure that the axis of the measuring device and the axis of deep-hole parts are located on the same straight line. Moreover, the distance between the supporting arm and the center of measuring device is slightly larger than the 
radius of the measured hole when the compression spring in the unit is in a free state. Thus, the compression spring is always in a compressed state when the measuring device works, ensuring that the universal ball at the end of the supporting arm is always in close contact with the wall surface. According to this feature, the developed device is suitable for measuring deep-hole parts with a diameter of 150 $160 \mathrm{~mm}$. A three-dimensional model and a solid model of the measuring device are exhibited in Fig.10 and Fig.11, respectively.

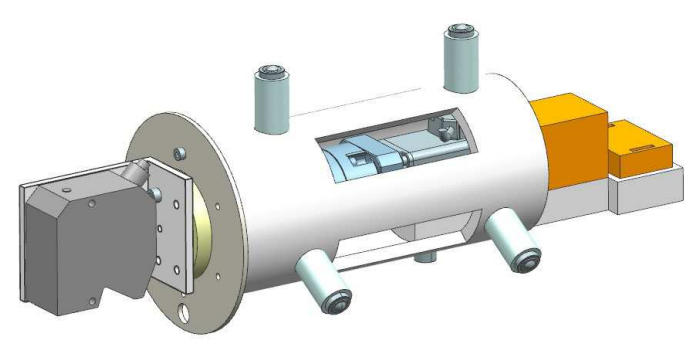

Fig.10 Three-dimensional model of measuring device.

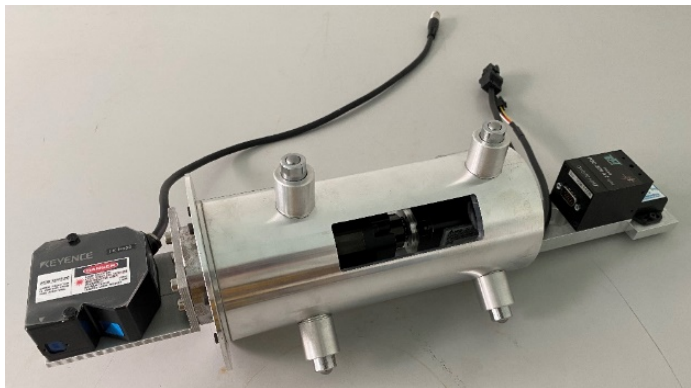

Fig.11 Solid model of measuring device.

The measuring device completes the movement and sampling under the control of signals and transmits the data to the computer for subsequent processing. On this basis, the computer software platform was established using Matlab language, as illustrated in Fig.12. The main functions are:

(1) Output the signal to perform pulse control on servo motor such as start-stop, clockwise, and counterclockwise rotation;

(2) Control the sensors to complete the collection, storage and transmission of measurement data;

(3) Obtain roundness and straightness errors by performing the coordinate transformation, section center calculation, roundness evaluation, and straightness evaluation on the sensor measurement data.

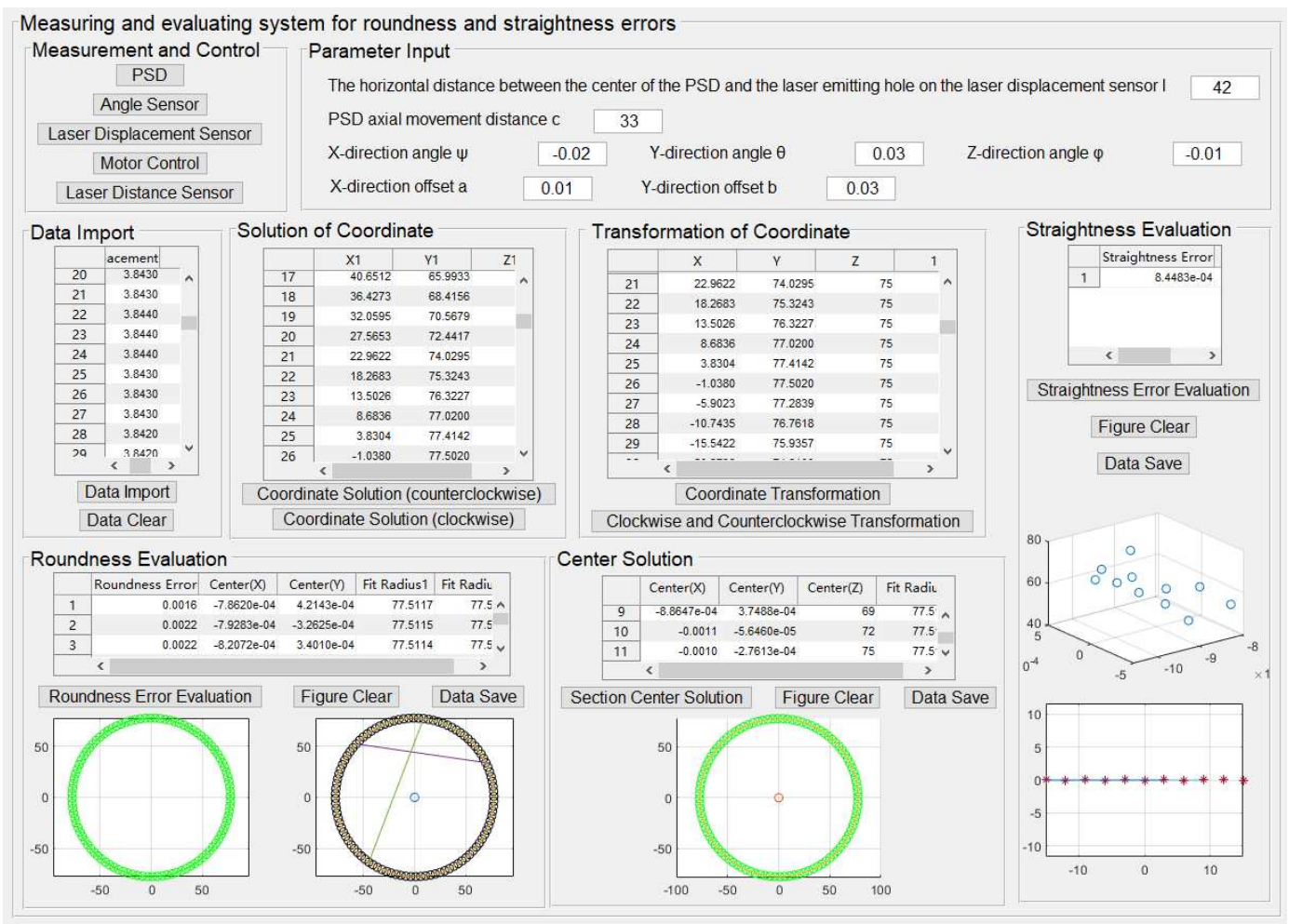

\section{Experimental research}

Fig.12 The computer software platform. 


\subsection{Calibration experiment of a laser displacement sensor}

There is an offset distance $a$ and an eccentricity value $b$ between the laser emission hole and the rotating axis (Fig.13). Therefore, the distance between each sampling point and the rotating center is not equal to the output value of the laser displacement sensor, making parameters $a$ and $b$ exert a huge influence on the measurement results. It is necessary to eliminate the errors caused by parameters $a$ and $b$ in the measurement process by calibrating them.

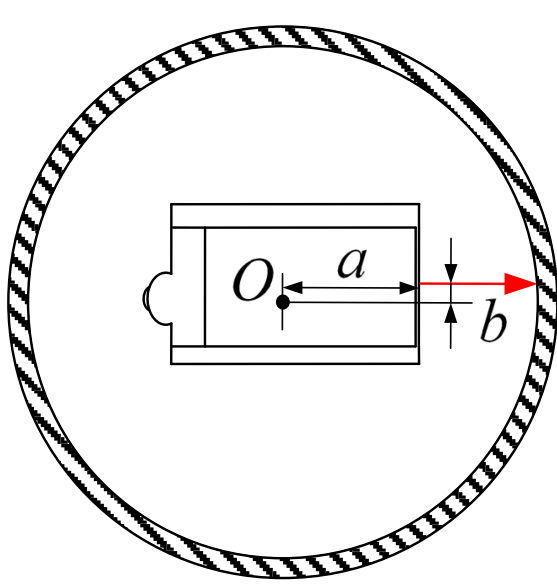

Fig. 13 Schematic diagram of geometric

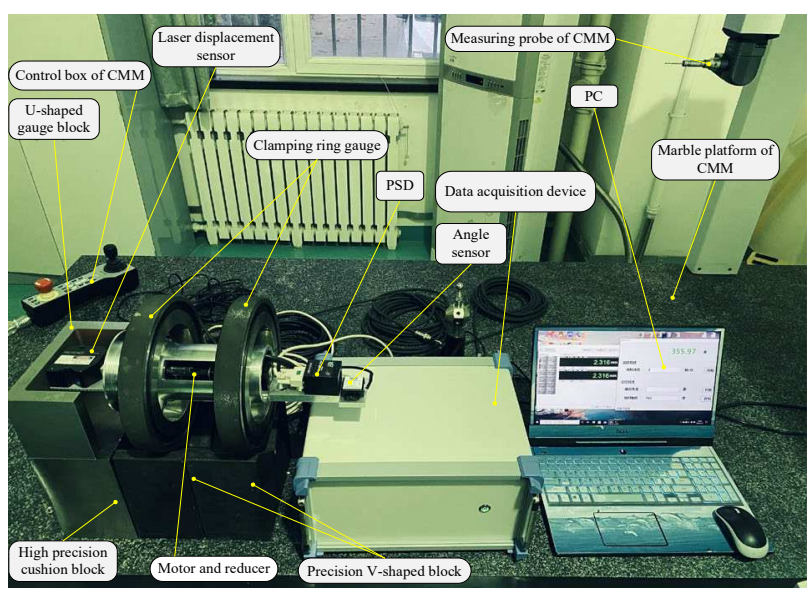

Fig. 14 The calibration experiment scene.

parameters of a laser displacement sensor.

A pair of precision V-shaped blocks and a high-precision cushion block were placed on the marble platform of the CMM. By moving the measuring probe of CMM and adjusting the positions of the precision V-shaped blocks and high-precision cushion block, their front surfaces were located on the same plane. Then, two groups of supporting arms of the measuring device were placed in two clamping ring gauges with inner diameters of $155 \mathrm{~mm}$, respectively, and the two clamping ring gauges were stuck and fixed on the precision V-shaped blocks, so as to simulate the fixed mode of the measuring device in the deep-hole parts. Then, a U-shaped gauge block with good flatness and verticality was placed on the cushion block, and the lower surface of the U-shaped gauge block and the side surface of the cushion block were located on the same plane using the measuring probe of CMM. Afterward, the motor was rotated to make the lower bottom face of the mounting bracket for fixing the laser displacement sensor face upwards. The measuring probe of CMM and the motor were used to make a fine adjustment to keep the lower bottom face of the mounting bracket horizontal, so as to determine the position where the laser emitted by the laser displacement sensor was perpendicular to the calibration plane. The experimental scene is displayed in Fig.14.

Parameters $a$ and $b$ were calibrated on the section passing through the laser and perpendicular to the rotating axis. The calibration principle of parameter $a$ is presented in Fig.15. The laser displacement sensor was rotated to face the calibration plane A, the position of the sensor was adjusted to make the laser perpendicular to the plane, and the output value $r_{1}$ of the sensor was recorded. Then, the sensor was rotated to face the calibration plane $\mathrm{B}$, the position of the sensor was adjusted to make the laser perpendicular to the plane, and the output value $r_{2}$ was recorded. According to the geometric relationship, the offset distance $a$ between the rotating axis and the laser emitting surface of the laser displacement sensor can be calculated by Eq. (9).

$$
a=\frac{\left(D-r_{1}-r_{2}\right)}{2}
$$

where $D$ denotes the calibration distance of U-shaped gauge block. 


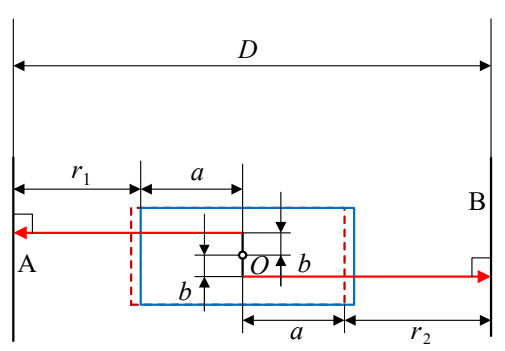

Fig.15 The calibration principle of parameter $a$.

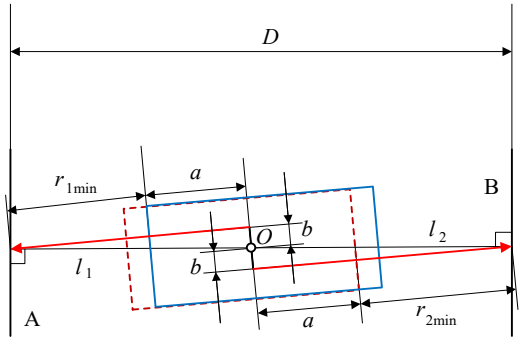

Fig.16 The calibration principle of parameter $b$.

The calibration principle of parameter $b$ is illustrated in Fig.16. Specifically, the laser displacement sensor was rotated to face the calibration plane A, the position of the sensor was adjusted within the angle of \pm 15 between the laser and the plane, and the minimum output value $r_{1 \mathrm{~min}}$ of the sensor was recorded. Since the parameters $a$ and $b$ are fixed values, the value $l_{1}$ corresponding to the value $r_{1 \text { min }}$ is the minimum, suggesting that the straight line where $l_{1}$ lies is perpendicular to the plane $\mathrm{A}$. Then, the laser displacement sensor was rotated to face the calibration plane $B$, the position of the sensor was adjusted within the angle of \pm 15 between the laser and the plane, and the minimum output value $r_{2 \min }$ of the sensor was recorded. Similarly, the value $l_{2}$ corresponding to the value $r_{2 \min }$ is the minimum, and the straight line where $l_{2}$ lies is perpendicular to plane $\mathrm{B}$. According to the geometric relationship, the eccentricity value $b$ can be calculated by Eq. (10).

$$
\left\{\begin{array}{l}
l_{1}+l_{2}=D \\
l_{1}=\sqrt{\left(a+r_{1 \text { min }}\right)^{2}+b^{2}} \\
l_{2}=\sqrt{\left(a+r_{2 \text { min }}\right)^{2}+b^{2}}
\end{array}\right.
$$

The U-shaped gauge blocks were changed with different calibration distances, and parameters $a$ and $b$ were calibrated five times, so as to reduce the calibration error. Among them, the calibration distance of U-shaped gauge blocks can be measured by CMM. The calibration results of the parameters $a$ and $b$ are provided in Table 1 and Table 2, respectively. The average value of five measurement results is selected to obtain $a=31.345 \mathrm{~mm}$ and $b=1.038 \mathrm{~mm}$.

Table 1 The calibration results of parameter $a$.

\begin{tabular}{|c|c|c|c|c|c|c|}
\hline Number & $\begin{array}{c}\text { Standard value } \\
D(\mathrm{~mm})\end{array}$ & $\begin{array}{c}\text { Value of CMM } \\
\qquad D_{1}(\mathrm{~mm})\end{array}$ & $\begin{array}{c}r_{1} \\
(\mathrm{~mm})\end{array}$ & $\begin{array}{c}r_{2} \\
(\mathrm{~mm})\end{array}$ & $\begin{array}{c}a \\
(\mathrm{~mm})\end{array}$ & $\begin{array}{c}\text { Average value } \\
(\mathrm{mm})\end{array}$ \\
\hline 1 & 150 & 150.002 & 44.769 & 42.535 & 31.349 & \multirow{5}{*}{31.345} \\
\hline 2 & 152 & 152.004 & 46.355 & 42.967 & 31.341 & \\
\hline 3 & 154 & 154.006 & 47.450 & 43.866 & 31.345 & \\
\hline 4 & 156 & 156.003 & 48.710 & 44.597 & 31.348 & \\
\hline 5 & 158 & 158.003 & 49.090 & 46.227 & 31.343 & \\
\hline
\end{tabular}

Table 2 The calibration results of parameter $b$.

\begin{tabular}{|c|c|c|c|c|c|c|}
\hline Number & $\begin{array}{c}\text { Nominal value } \\
\qquad D(\mathrm{~mm})\end{array}$ & $\begin{array}{c}\text { Value of CMM } \\
\qquad D_{l}(\mathrm{~mm})\end{array}$ & $\begin{array}{l}r_{\min } \\
(\mathrm{mm})\end{array}$ & $\begin{array}{l}r_{2 \min } \\
(\mathrm{mm})\end{array}$ & $\begin{array}{c}b \\
(\mathrm{~mm})\end{array}$ & $\begin{array}{c}\text { Average value } \\
\qquad(\mathrm{mm})\end{array}$ \\
\hline 1 & 150 & 150.002 & 44.766 & 42.532 & 1.024 & \multirow{5}{*}{1.038} \\
\hline 2 & 152 & 152.004 & 46.344 & 42.956 & 1.031 & \\
\hline 3 & 154 & 154.006 & 47.441 & 43.861 & 1.037 & \\
\hline 4 & 156 & 156.003 & 48.708 & 44.594 & 1.045 & \\
\hline 5 & 158 & 158.003 & 49.080 & 46.219 & 1.051 & \\
\hline
\end{tabular}




\subsection{Measurement and evaluation experiment of roundness and straightness errors}

To verify the effectiveness of the proposed measuring device and evaluation method, the roundness and straightness errors of the measuring ring gauge with an inner diameter of $155 \mathrm{~mm}$, the thickness of $36 \mathrm{~mm}$, nominal roundness error of $2.5 \mu \mathrm{m}$, and nominal straightness error of $3 \mu \mathrm{m}$ were measured and evaluated using the CMM and the developed system, respectively. The results were compared and analyzed.

The inner hole of the measuring ring gauge was equally divided into 13 sections every $3 \mathrm{~mm}$ along the thickness direction. The first and last sections were removed, with a total of 11 sections. Besides, the inner hole surface was scanned and equiangularly sampled using the Hexagon Global Advantage CMM. The number of sampling points in each section is 100 . Afterward, the shape error was evaluated according to the least square method equipped by the CMM. The measuring ambient temperature is $20.2^{\circ} \mathrm{C}$, and the relative humidity is $35 \%$. Furthermore, three groups of experiments were performed on three measuring ring gauges of the same model to eliminate the experimental contingency. The measurement and evaluation results are listed in Table 3.

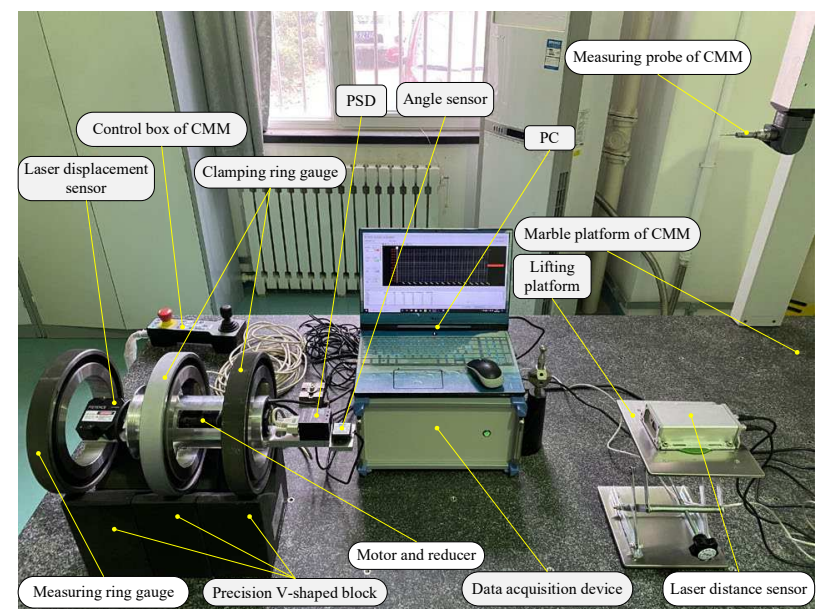

Fig. 17 The measurement experimental scene with developed device

Under the same measuring environment, three identical precision V-shaped blocks were arranged side by side on the marble platform of CMM. Their front surfaces were located on the same plane by moving the measuring probe of CMM and adjusting the position of the precision V-shaped blocks. Then, two groups of supporting arms of the measuring device were placed in two clamping ring gauges with inner diameters of $155 \mathrm{~mm}$, respectively, so as to simulate the fixed mode of the measuring device in the deephole parts. The two clamping ring gauges were stuck and fixed on the precision V-shaped block. Besides, the measuring ring gauge was fixed on the front $\mathrm{V}$-shaped block, and the left side of the measuring ring gauge was made coplanar with the left side of the V-shaped block by the measuring probe of CMM. Particularly, the right side of the measuring ring gauge was taken as the measuring zero plane, and the measuring device was slowly moved until the laser line emitted by laser displacement sensor was in the measuring zero plane. Then, the installation position of the angle sensor was flattened by the measuring probe of CMM, and the angle sensor was zeroed and calibrated at this position. The motor was rotated to make the lower bottom of the mounting bracket for fixing the laser displacement sensor face upwards, and the measuring probe of CMM and the motor were used for fine adjustment to keep the lower bottom of the mounting bracket horizontal. Furthermore, the laser distance sensor located on the backside of the measuring device and fixed on the lifting platform was adjusted to allow the laser line emitted by the sensor vertically to irradiate the center of PSD photosensitive plane. Starting from the measuring zero 
plane, the measuring device was moved forward by $3 \mathrm{~mm}$ every time, and 11 measurement sections were obtained and the same as those in the CMM measuring experiment. The measurement experimental scene with the developed device is exhibited in Fig.17.

Table 3 The measurement and evaluation results of roundness and straightness errors.

\begin{tabular}{|c|c|c|c|c|c|c|c|}
\hline \multicolumn{2}{|c|}{ Measuring } & \multicolumn{3}{|c|}{ Roundness error $(\mathrm{mm})$} & \multicolumn{3}{|c|}{ Straightness error $(\mathrm{mm})$} \\
\hline $\begin{array}{l}\text { Ring } \\
\text { gauge }\end{array}$ & Section & Nominal & $\mathrm{CMM}$ & $\begin{array}{c}\text { Developed } \\
\text { device }\end{array}$ & Nominal & $\mathrm{CMM}$ & $\begin{array}{c}\text { Developed } \\
\text { device }\end{array}$ \\
\hline \multirow{11}{*}{1} & 1 & \multirow{11}{*}{0.0025} & 0.0018 & 0.0023 & \multirow{11}{*}{0.0030} & \multirow{11}{*}{0.0005} & \multirow{11}{*}{0.0017} \\
\hline & 2 & & 0.0015 & 0.0023 & & & \\
\hline & 3 & & 0.0015 & 0.0023 & & & \\
\hline & 4 & & 0.0014 & 0.0021 & & & \\
\hline & 5 & & 0.0016 & 0.0021 & & & \\
\hline & 6 & & 0.0015 & 0.0018 & & & \\
\hline & 7 & & 0.0014 & 0.0020 & & & \\
\hline & 8 & & 0.0013 & 0.0020 & & & \\
\hline & 9 & & 0.0013 & 0.0015 & & & \\
\hline & 10 & & 0.0024 & 0.0029 & & & \\
\hline & 11 & & 0.0021 & 0.0024 & & & \\
\hline \multirow{11}{*}{2} & 1 & \multirow{11}{*}{0.0025} & 0.0016 & 0.0016 & \multirow{11}{*}{0.0030} & \multirow{11}{*}{0.0003} & \multirow{11}{*}{0.0008} \\
\hline & 2 & & 0.0015 & 0.0022 & & & \\
\hline & 3 & & 0.0017 & 0.0022 & & & \\
\hline & 4 & & 0.0016 & 0.0022 & & & \\
\hline & 5 & & 0.0016 & 0.0020 & & & \\
\hline & 6 & & 0.0015 & 0.0020 & & & \\
\hline & 7 & & 0.0015 & 0.0020 & & & \\
\hline & 8 & & 0.0016 & 0.0020 & & & \\
\hline & 9 & & 0.0018 & 0.0022 & & & \\
\hline & 10 & & 0.0019 & 0.0023 & & & \\
\hline & 11 & & 0.0020 & 0.0027 & & & \\
\hline \multirow{11}{*}{3} & 1 & \multirow{11}{*}{0.0025} & 0.0018 & 0.0023 & \multirow{11}{*}{0.0030} & \multirow{11}{*}{0.0003} & \multirow{11}{*}{0.0011} \\
\hline & 2 & & 0.0017 & 0.0022 & & & \\
\hline & 3 & & 0.0017 & 0.0022 & & & \\
\hline & 4 & & 0.0016 & 0.0020 & & & \\
\hline & 5 & & 0.0016 & 0.0019 & & & \\
\hline & 6 & & 0.0017 & 0.0020 & & & \\
\hline & 7 & & 0.0018 & 0.0018 & & & \\
\hline & 8 & & 0.0018 & 0.0020 & & & \\
\hline & 9 & & 0.0018 & 0.0018 & & & \\
\hline & 10 & & 0.0017 & 0.0022 & & & \\
\hline & 11 & & 0.0018 & 0.0023 & & & \\
\hline
\end{tabular}

The sampling frequency of the laser displacement sensor was set to $1 \mathrm{kHz}$, and the storage period was 1000 times of the sampling period, that is, one second recorded the data of one point. Since the number of measuring points in each section is 100 , the motor speed should be set to $0.6 \mathrm{rpm}$. When measuring, 
in the first section, the horizontal direction was taken as the starting position, counterclockwise rotation was performed, and sampling was conducted every $3.6^{\circ}$. To prevent the missing of measurement points caused by the backlash of the reducer during the change of the rotation direction, each sampling was rotated by an additional $15^{\circ}$, and the redundant sampling points were removed during data processing. On the second section, starting from the end position of the first measurement process, the sampling started after rotating clockwise by $15^{\circ}$; it also rotated by an additional $15^{\circ}$ at the end. By analogy, counterclockwise and clockwise rotation was alternately conducted to avoid the winding of cables during the measurement process, realizing the measurement of 11 sections. The information collected by the sensors was transmitted to the PC through the data acquisition device. Finally, the roundness and straightness errors were evaluated by the improved MZC method and the rotating projection method. Similarly, three measuring ring gauges were tested, as indicated in Table 3.
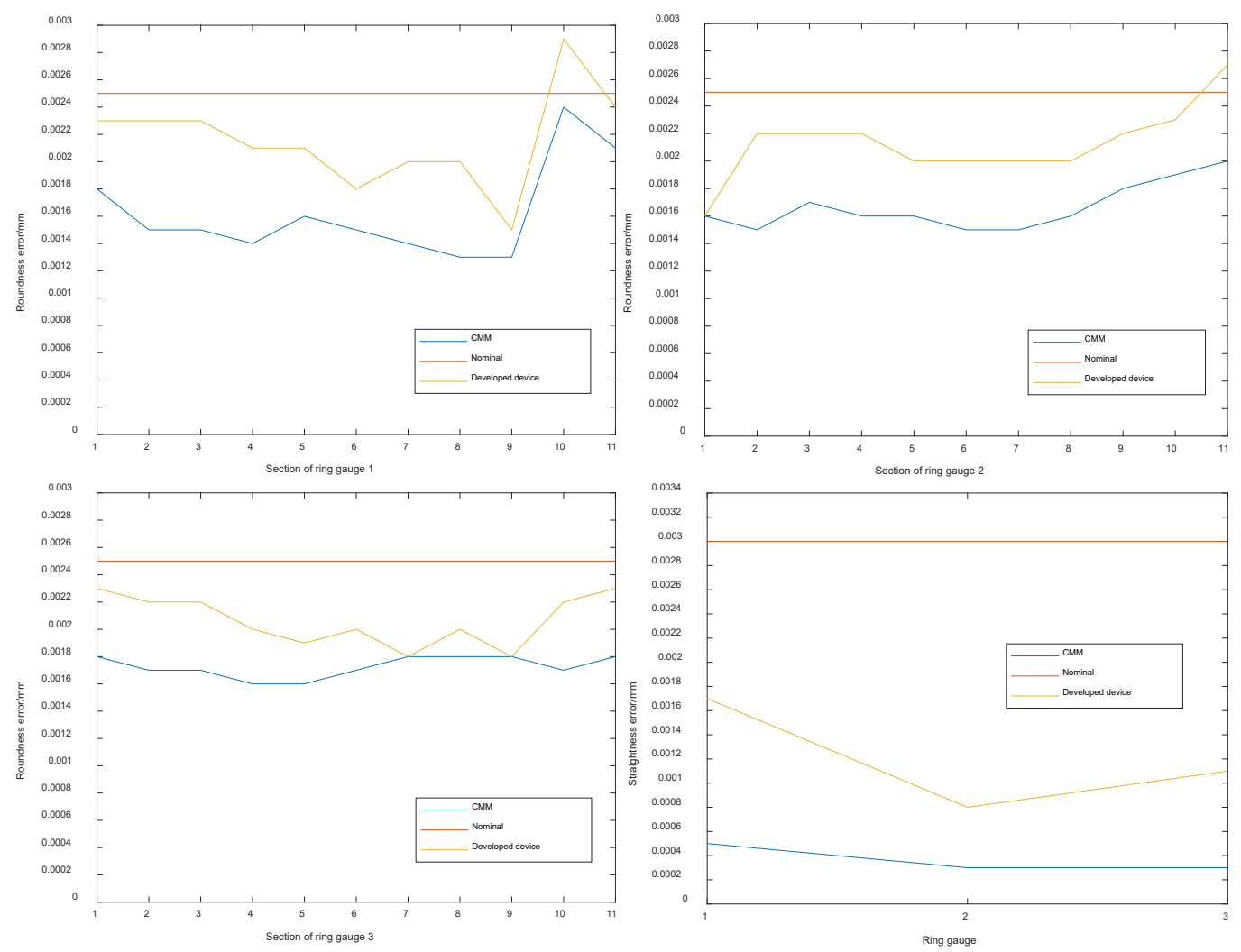

Fig. 18 Comparison of measurement results.

The results demonstrate that the roundness and straightness error evaluation values of each measured section of the three measuring ring gauges are within the nominal value range under the mode of being measured by CMM and evaluated by the least square method. Compared with this, the developed device is used to measure the measuring ring gauge in a non-contact way. The improved MZC method and the rotating projection method based on the minimum zone principle are used to evaluate the roundness and straightness errors. Although all the evaluation values increase to some extent, except for the roundness evaluation values of the 10th measuring section of ring gauge 1 and the 11th measuring section of ring gauge 2, the remaining results are all within the nominal value range. As shown in Fig. 18, we can see that the measurement result curve of the developed device is much closer to the nominal value curve than the CMM measurement result curve, that is to say, the developed system shows a better measurement and evaluation effect. Therefore, the validity and accuracy of the measuring device and the evaluation method adopted are verified. 


\section{Conclusion}

According to the measuring principle of roundness and straightness errors of deep-hole parts, an integrated measuring device composed of laser displacement sensor, angle sensor, PSD, and laser distance sensor is developed, which is suitable for measuring deep-hole parts with a diameter of 150$160 \mathrm{~mm}$. Based on the distance relationship between the measurement point set and the center point and the polygon removal rule, the control points of MCC and MIC are searched to construct the MZC, avoid the complexity of solving the inner and outer convex hull point sets and the evaluation error caused by the uniqueness of the inner convex hull point set, and optimize the solving process of the control points. Then, the improved MZC method proposed in this paper and the rotation projection method based on the minimum area principle proposed by Chen Hui are applied to the evaluation process of roundness and straightness errors, respectively. Finally, comparing with the measurement results of CMM, the measurement results of developed device are much closer to the nominal value, verifying the effectiveness and accuracy of the roundness and straightness error measuring device and roundness error evaluation method for deep-hole parts. The developed measurement and evaluation mode can be used in the field of high-precision measurement, and its accuracy can reach the micron level according to experiments.

\section{Acknowledgements}

This work is supported by the National Natural Science Foundation of China (grant no. 52075042).

\section{References}

[1] Salah E, Junghyuk K, Martin J. Micro-scale hole profile measurement using rotating wire probe and acoustic emission contact detection[J]. Measurement, 2016, 89:215-222.

[2] Chang T Y, Liao Y S, Liu W C. Development of a micro hole measuring system based on the capacitance principle[J]. Measurement Science \& Technology, 2009, 20(10):105104.

[3] Mekid S, Vacharanukul K. In-process out-of-roundness measurement probe for turned workpieces[J]. Measurement, 2011, 44(4):762-766.

[4] Ma Y Z, Yu Y X, Wang X H. Diameter measuring technique based on capacitive probe for deep hole or oblique hole monitoring[J]. Measurement, 2014, 47:42-44.

[5] Akio K, Takao S, Hiroshi M, et al. Development of a laser-guiding-type deep small-sized hole-measurement system: Measurement accuracy[J]. Precision Engineering, 2020, 63:18-32.

[6] Ma Y Z, Ma L, Zheng Y Z. The measurement techniques for angular 3-D pinholes based on capacitive probe[J]. Measurement, 2017, 97:145-148.

[7] Zatočilová A, Paloušek D, Brandejs J. Image-based measurement of the dimensions and of the axis straightness of hot forgings[J]. Measurement, 2016, 94:254-264.

[8] Jurevicius M, Skeivalas J, Urbanavicius R. Accuracy evaluation of two-dimensional straightness measurement method based on optical meter[J]. Measurement, 2013, 46(2):960-963.

[9] Vekteris V, Jurevicius M, Strishka V. Two-dimensional straightness measurement using optical meter[J]. Optical Engineering, 2008, 47:123605.

[10] Vekteris V, Jurevicius M, Turla V. Optical device for straightness measurement[J]. Applied Physics B, 2015, 121:203-208.

[11] Li X M, Shi Z Y. Application of convex hull in the assessment of roundness error[J]. International Journal of Machine Tools \& Manufacture, 2008, 48(6):711-714.

[12] Li X M, Liu H Q. A simple and efficient algorithm for evaluation of roundness error[J]. Measurement Science \& Technology, 2012, 23:087003. 
[13] Li X M, Zhang J C. A joint method for the maximum inscribed circle and minimum circumscribed circle[J]. Measurement, 2016, 87:189-193.

[14] Gadelmawla E S. Simple and efficient algorithms for roundness evaluation from the coordinate measurement data[J]. Measurement, 2010, 43(2):223-235.

[15] Lei X Q, Zhang C Y, Xue Y J, et al. Roundness error evaluation algorithm based on polar coordinate transform[J]. Measurement, 2011, 44(2):345-350.

[16] Goch G, Lübke K. Tschebyscheff approximation for the calculation of maximum inscribed/minimum circumscribed geometry elements and form deviations[J]. CIRP Annals-Manufacturing Technology, 2008, 57(1):517-520.

[17] Li X M, Shi Z Y. The relationship between the minimum zone circle and the maximum inscribed circle and the minimum circumscribed circle[J]. Precision Engineering, 2009, 33(3):284-290.

[18] Dhanish P B, Mathew J. A fast and simple algorithm for evaluation of minimum zone straightness error from coordinate data[J]. The International Journal of Advanced Manufacturing Technology, 2007, 32(1-2):92-98.

[19] Cho S, Kim J Y. Straightness and flatness evaluation using data envelopment analysis[J]. The International Journal of Advanced Manufacturing Technology, 2012, 63(5-8):731-740.

[20] Ding Y, Zhu L M, Ding H. Semidefinite programming for Chebyshev fitting of spatial straight line with applications to cutter location planning and tolerance evaluation[J]. Precision Engineering, 2007, 31(4):364368.

[21] Ding Y, Zhu L M, Ding H. A unified approach for circularity and spatial straightness evaluation using semidefinite programming[J]. International Journal of Machine Tools \& Manufacture, 2007, 47(10):1646-1650.

[22] Calvo R, Gómez E, Domingo R. Vectorial method of minimum zone tolerance for flatness, straightness, and their uncertainty estimation[J]. International Journal of Precision Engineering and Manufacturing, 2014, 15(1):31-44.

[23] Endrias D H, Feng H Y, MA J, et al. A combinatorial optimization approach for evaluating minimum-zone spatial straightness errors[J]. Measurement, 2012, 45(5):1170-1179.

[24] Rhinithaa P T, Selvakumar P, Nikhil S, et al. Comparative study of roundness evaluation algorithms for coordinate measurement and form data[J]. Precision Engineering, 2018, 51:458-467.

[25] Gadelmawla E S. Simple and efficient algorithms for roundness evaluation from the coordinate measurement data[J]. Measurement, 2010, 43(2):223-235.

[26] Chen H, Liu Z B, Wang X B. Evaluation of axis straightness error of hole parts by rotating projection method[J]. Journal of Harbin Institude of Technology, 2020, 52: 147-152. 


\section{Declaration}

1、Ethical Approval: Not applicable.

2、Consent to Participate: Not applicable.

3、Consent to Publish: Not applicable.

\section{4、Authors Contributions:}

A multi-sensor integrated device that can measure two kinds of shape errors simultaneously was developed. Aiming at the problem of roundness error evaluation, the solution process of the control points of the minimum zone circle was optimized by calculating the distance between points and searching according to the polygon removal rule. Besides, the rotating projection method was used to evaluate the straightness error effectively. Eventually, the effectiveness of the measuring device and the shape error evaluation method was verified by experimental research.

\section{5、 Funding:}

This work is supported by the National Natural Science Foundation of China (grant no. 52075042).

\section{6、Competing Interests:}

囚The authors declare that they have no known competing financial interests or personal relationships that could have appeared to influence the work reported in this paper.

$\square$ The authors declare the following financial interests/personal relationships which may be considered as potential competing interests:

\section{7、Availability of data and materials:}

The data sets and materials supporting the results of this article are included within the article. 
Figures

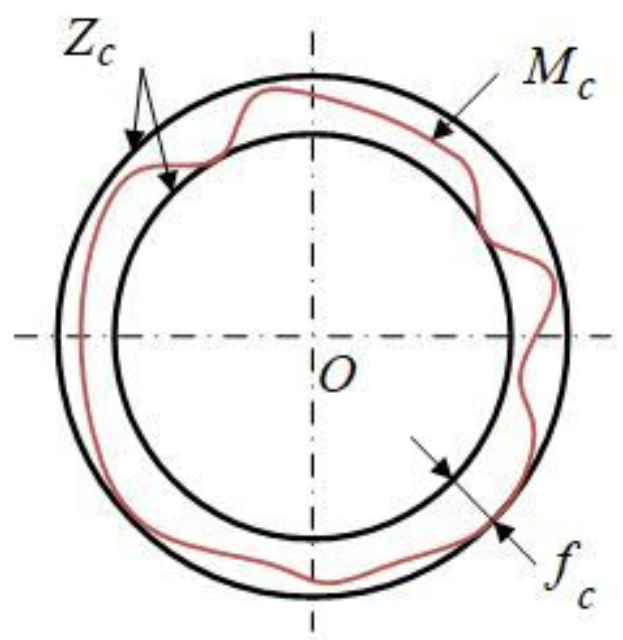

Figure 1

Schematic diagram of roundness error.

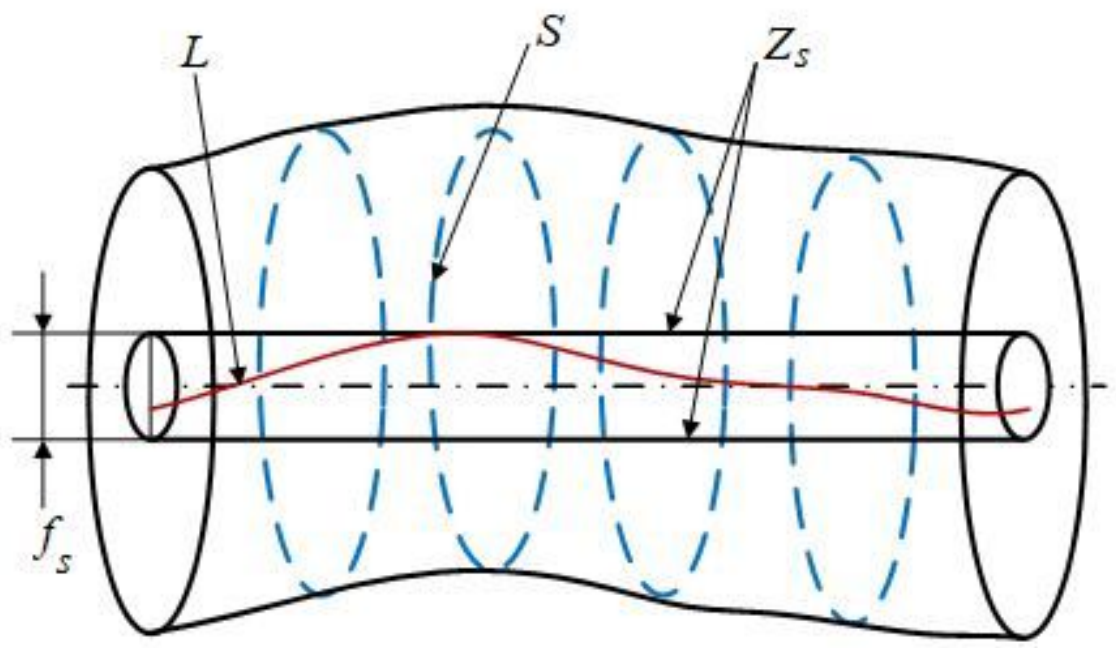

Figure 2

Schematic diagram of straightness error. 


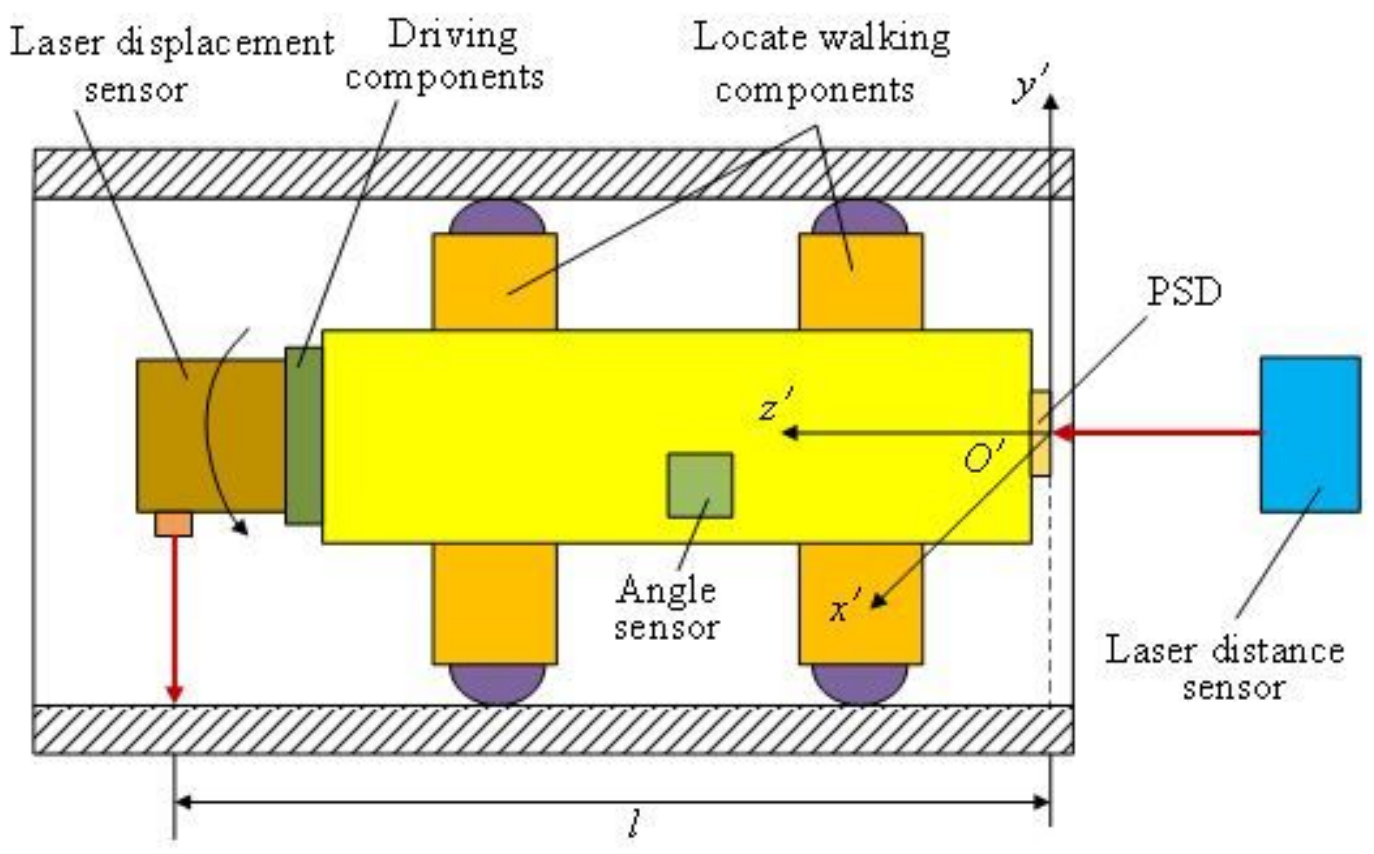

Figure 3

Schematic diagram of the measuring device.

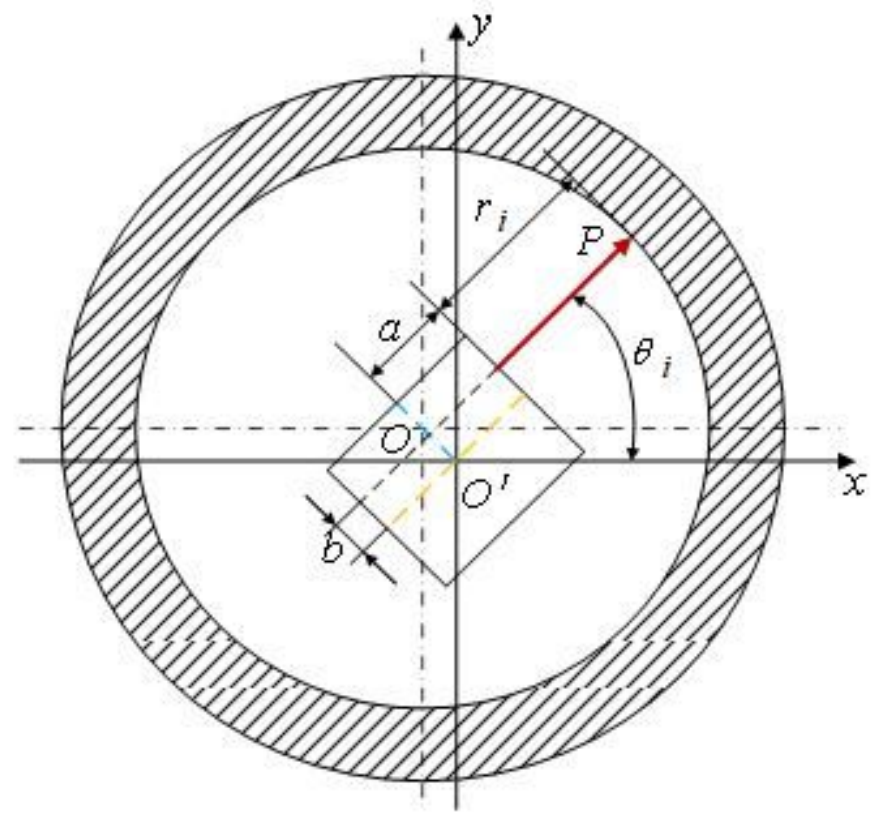

Figure 4

Schematic diagram of transformation from displacement value to coordinate value. 


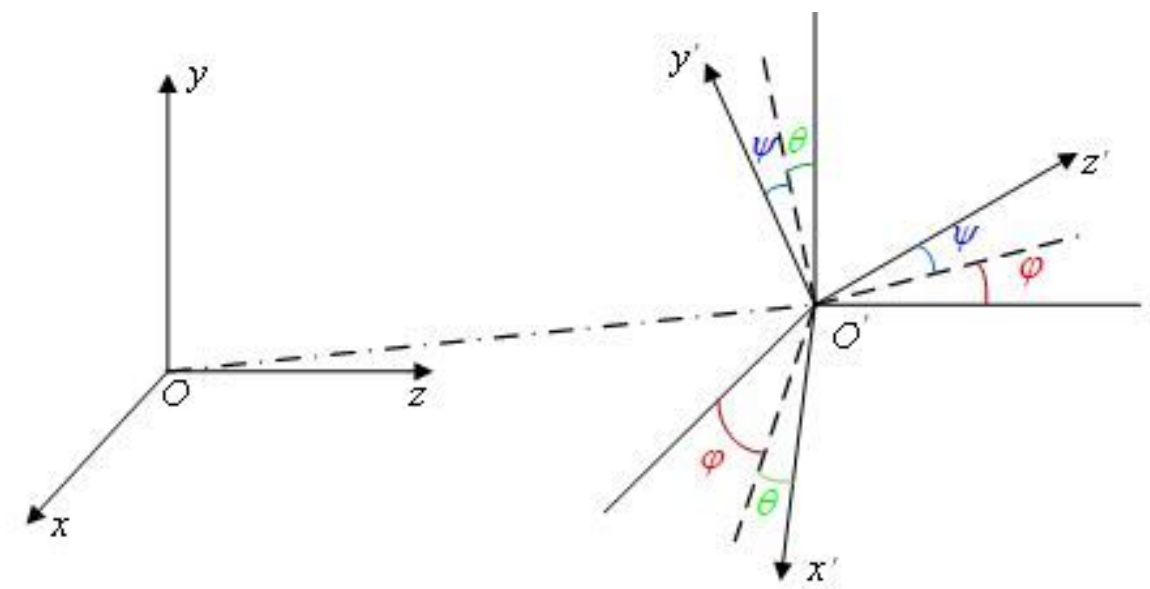

\section{Figure 5}

Transformation from measurement coordinate system to absolute coordinate system.

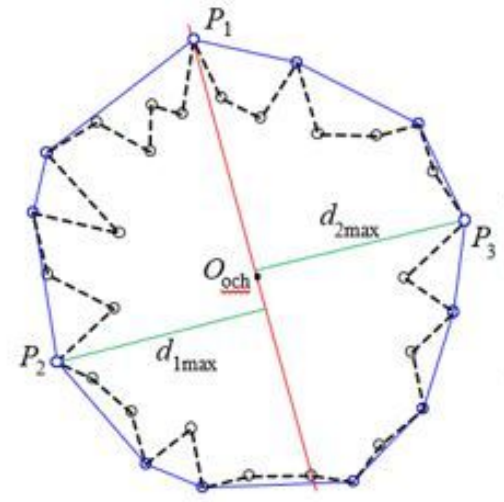

(a)

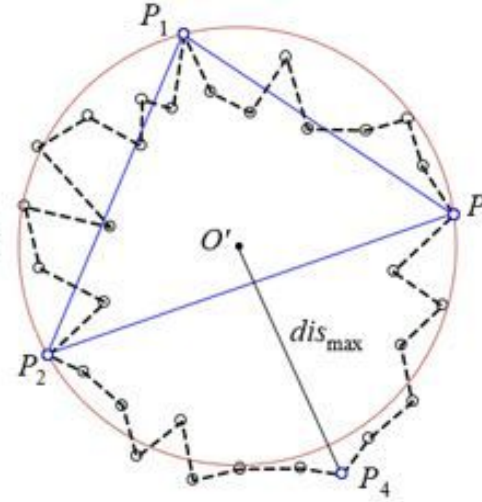

(b)

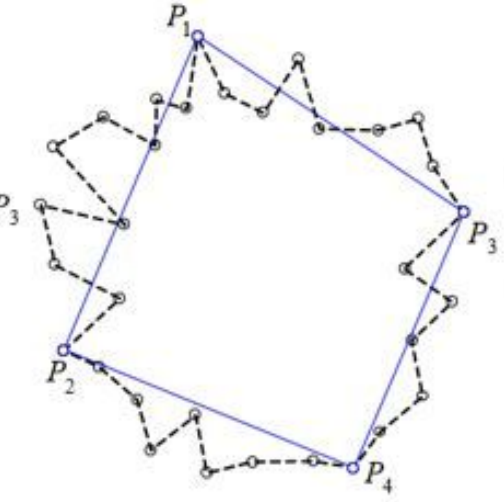

(c)

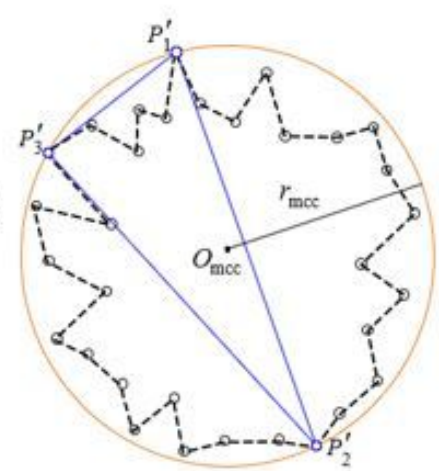

(d)

\section{Figure 6}

(a) Search the points P2 and P3 on both sides of the line P10och with the largest distance from it; (b) Search the point P4 with the largest distance from the center O' of MCC; (c) The point P4 is added into the triangle to form a quadrilateral; (d) The control points of MCC.

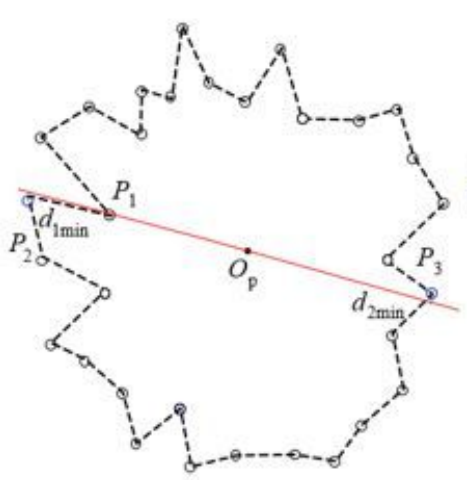

(a)

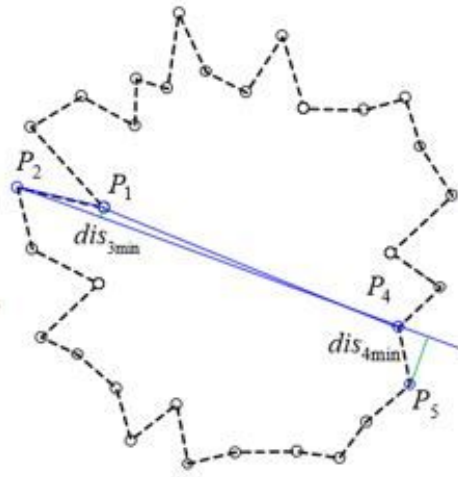

(b)

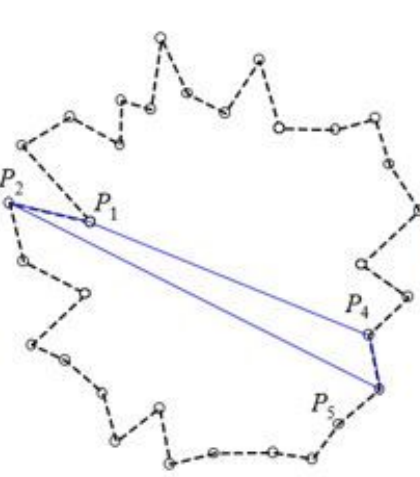

(c)

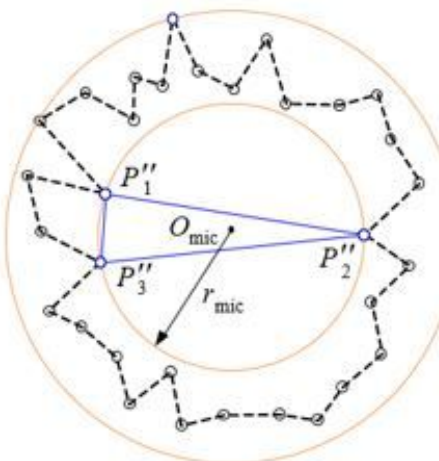

(d)

Figure 7 
(a) Search that points P2 and P3 on both side of P10p with the shortest distance from P10p; (b) Search the points P1 and P5 on both sides of the longest side P2P4 with the shortest distance from it; (c) The points P1 and P5 form a quadrilateral with P2 and P4; (d) The control point of the MIC.

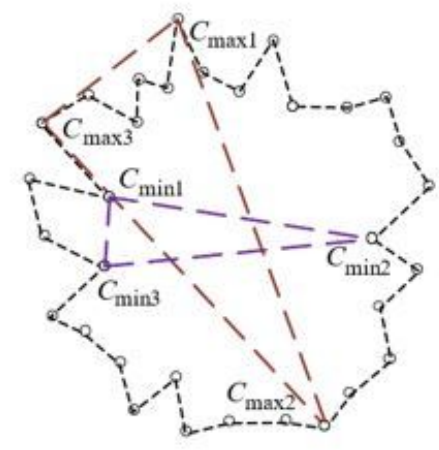

(a)

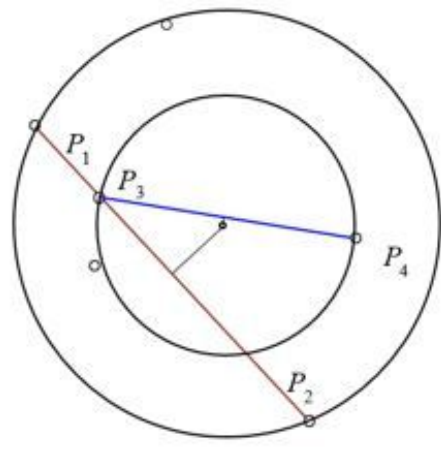

(b)

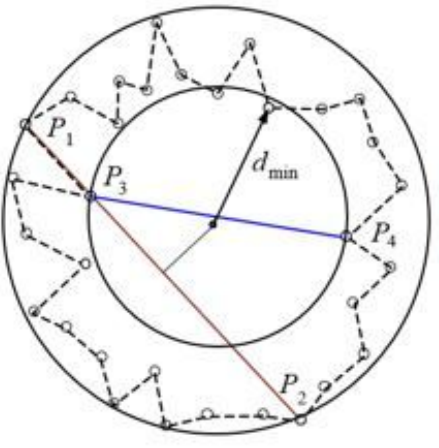

(c)

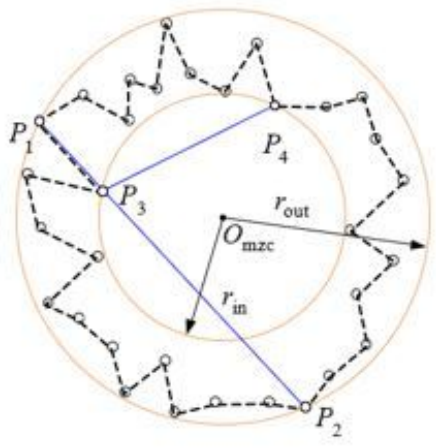

(d)

Figure 8

(a) The internal and external control points; (b) The concentric circle constructed contains internal and external control points; (c) The point corresponding to dmax is added into Dmax; (d) Roundness error evaluation (4) Roundness error evaluation

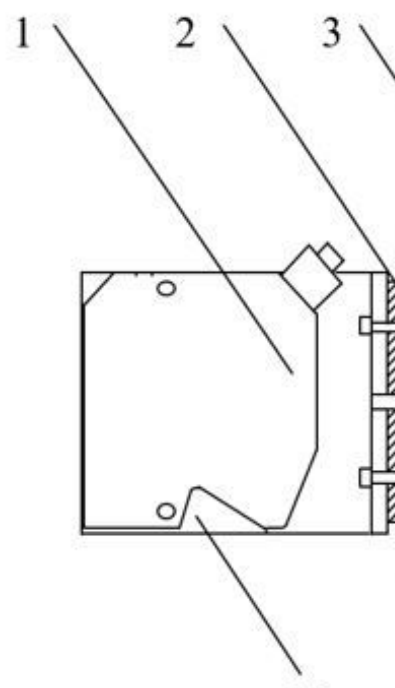

16

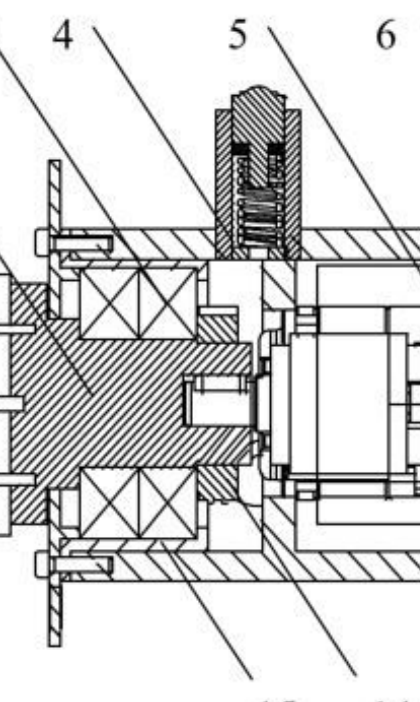

15

14

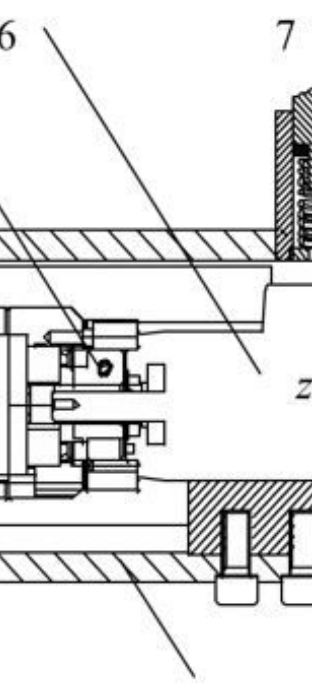

13
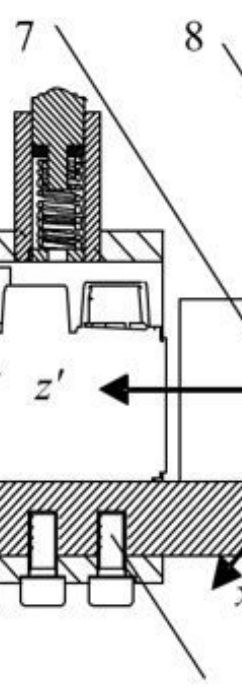

12

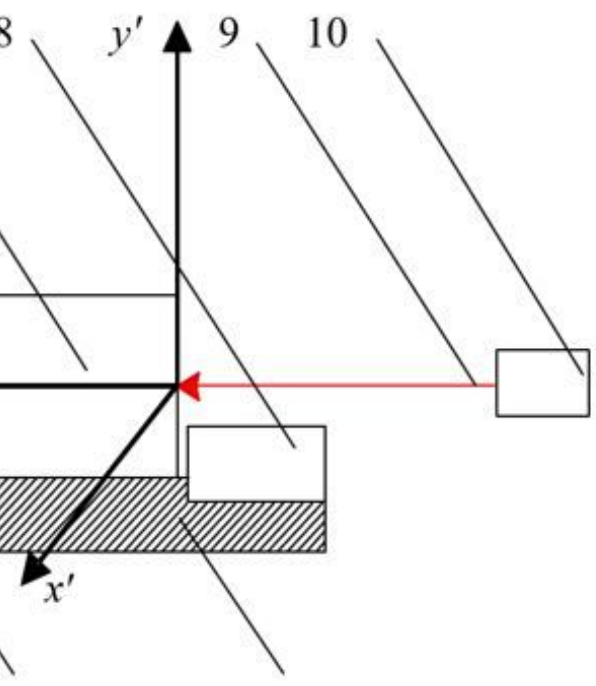

11

\section{Figure 9}

1-Laser displacement sensor, 2- Rotating shaft, 3-Angular contact ball bearing set, 4-Supporting arm, 5Reducer, 6-Servo motor, 7-Position sensitive detector, 8-Angle sensor, 9-Laser, 10-Laser distance sensor, 11-Motor supporting block, 12-Screw, 13-Outer cylinder, 14-Bearing lock nut, 15-Sleeve, 16-Mounting bracket Fig.9 Overall structure diagram of measuring device. 


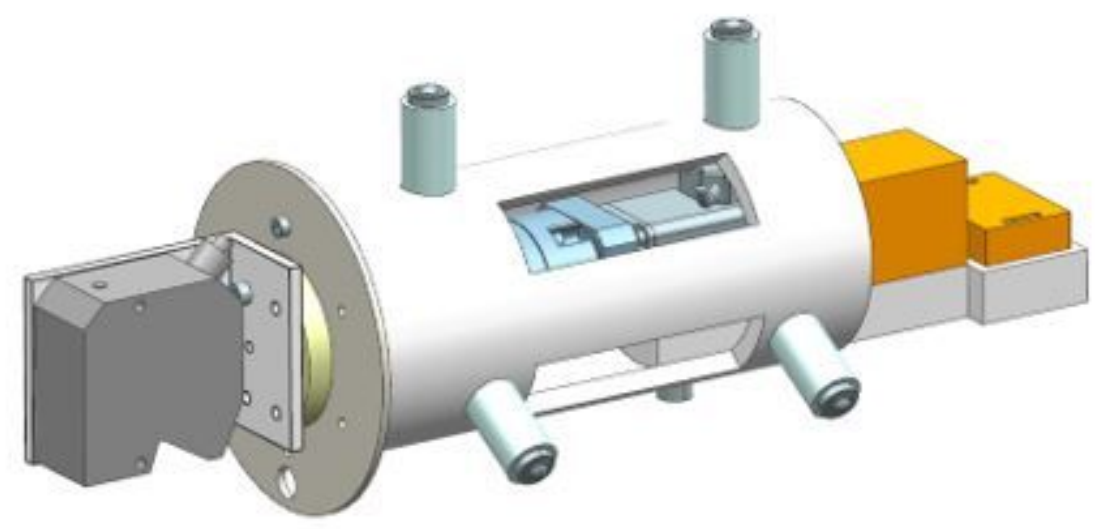

Figure 10

Three-dimensional model of measuring device.

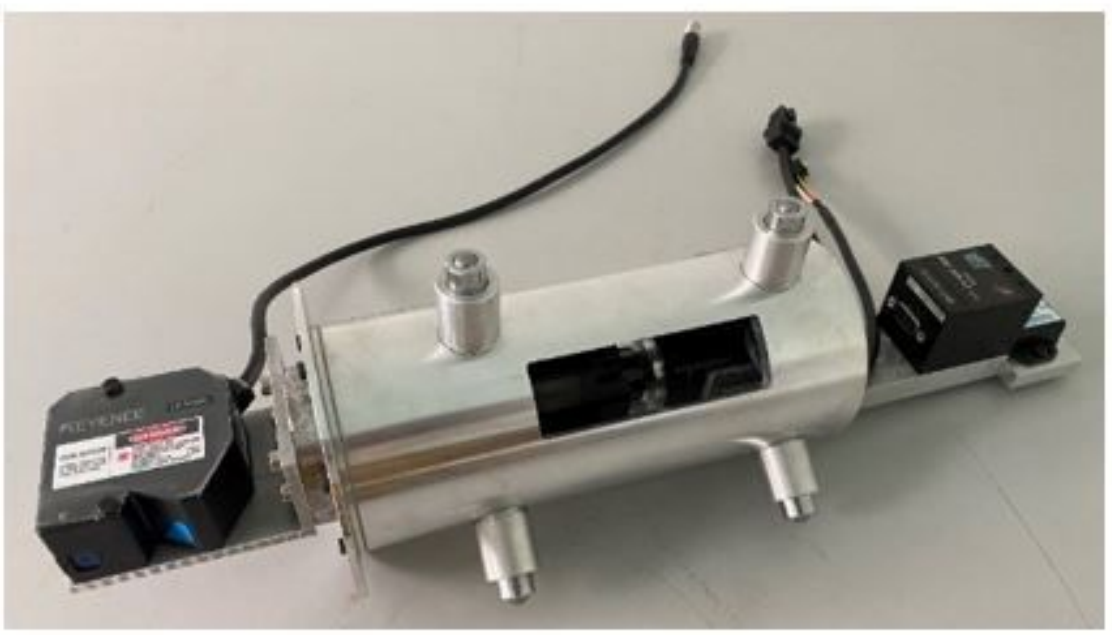

Figure 11

Solid model of measuring device. 


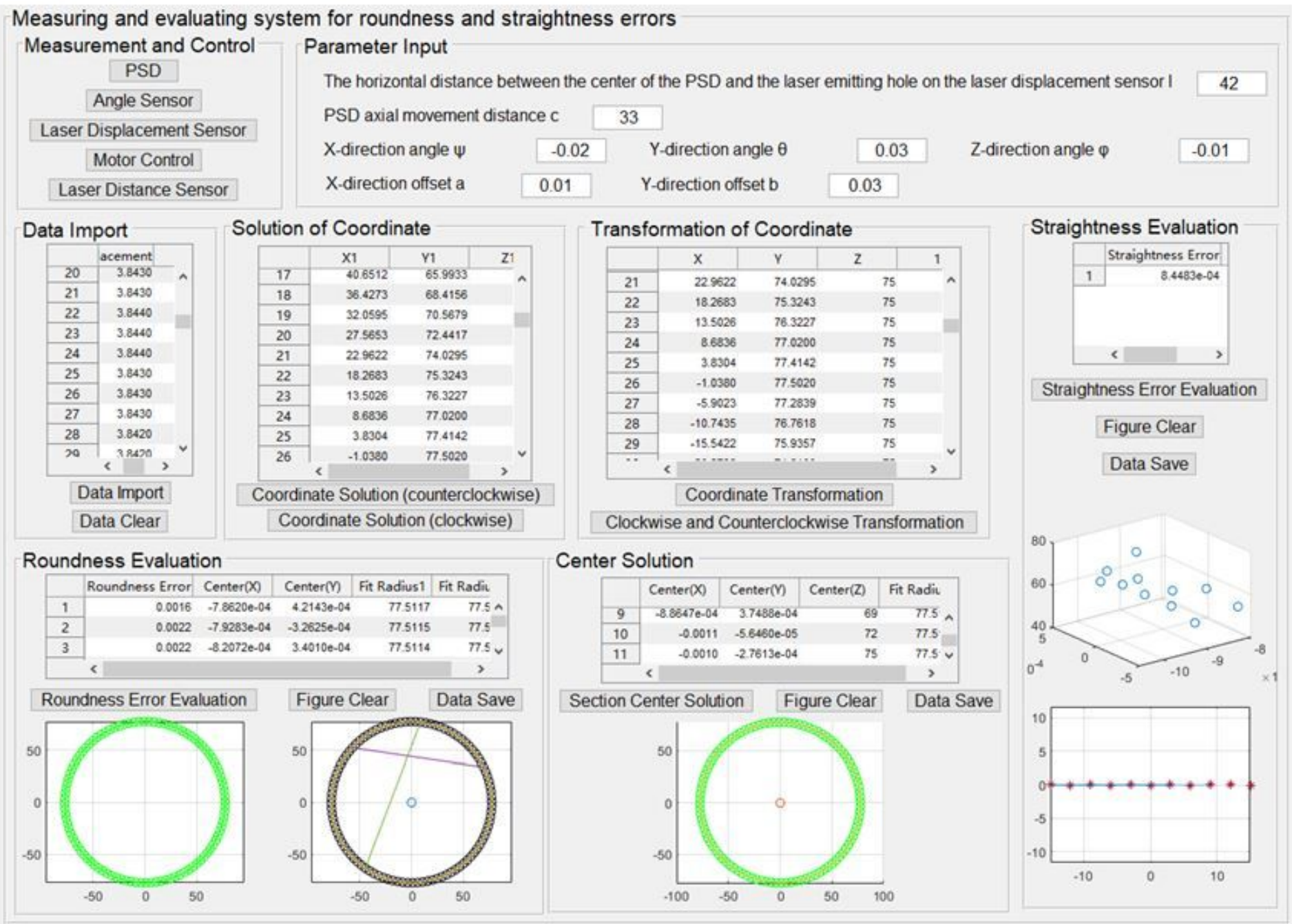

\section{Figure 12}

The computer software platform. 


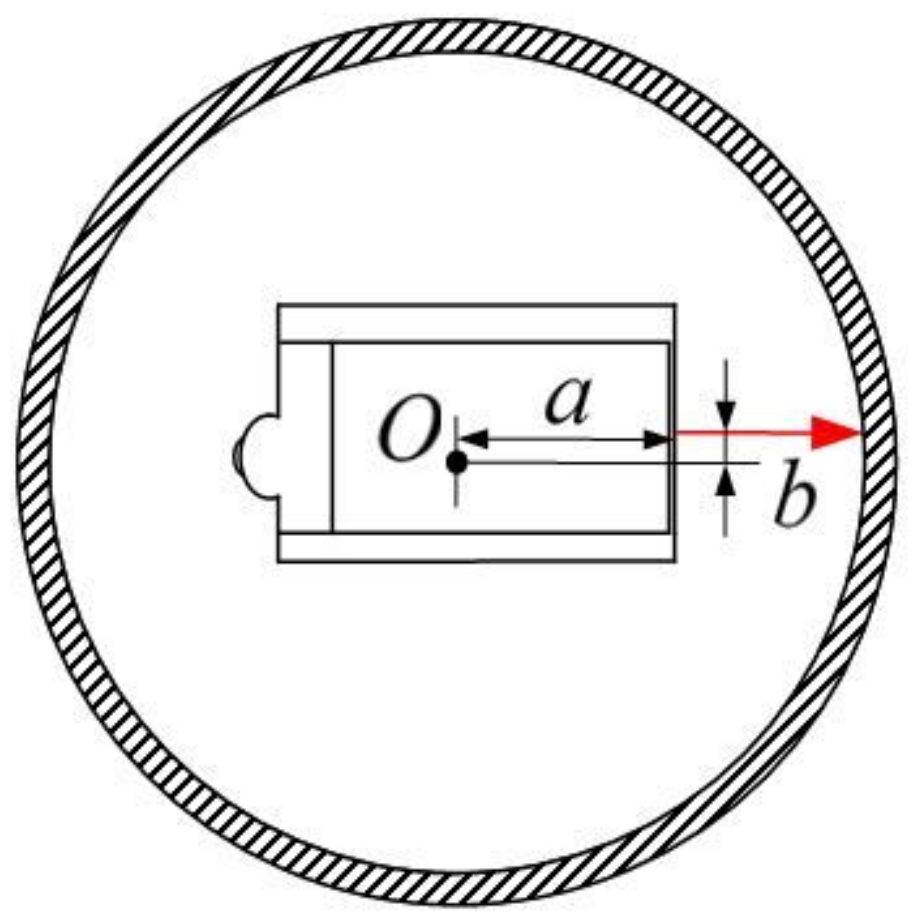

Figure 13

Schematic diagram of geometric parameters of a laser displacement sensor.

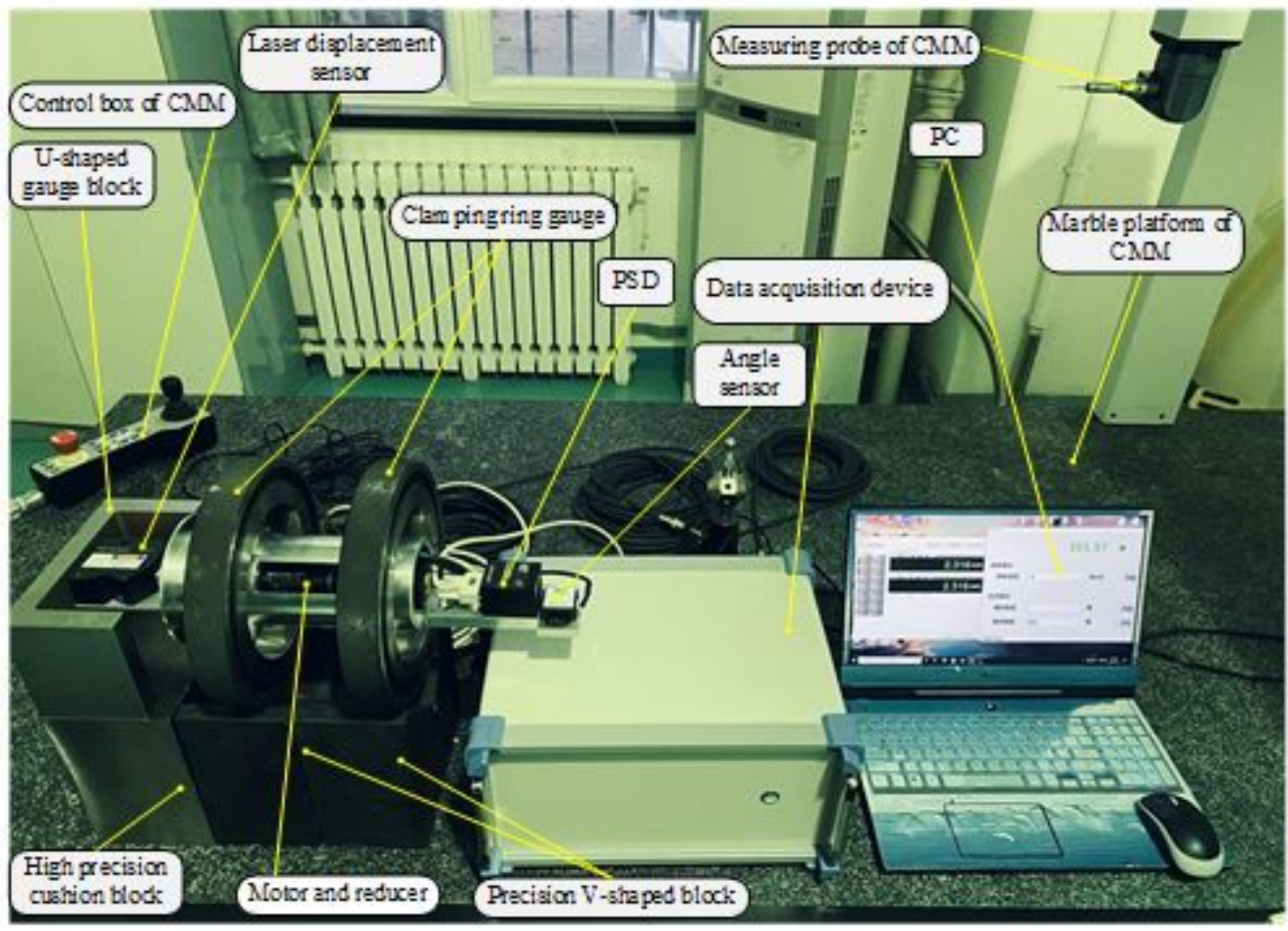

Figure 14

The calibration experiment scene. 


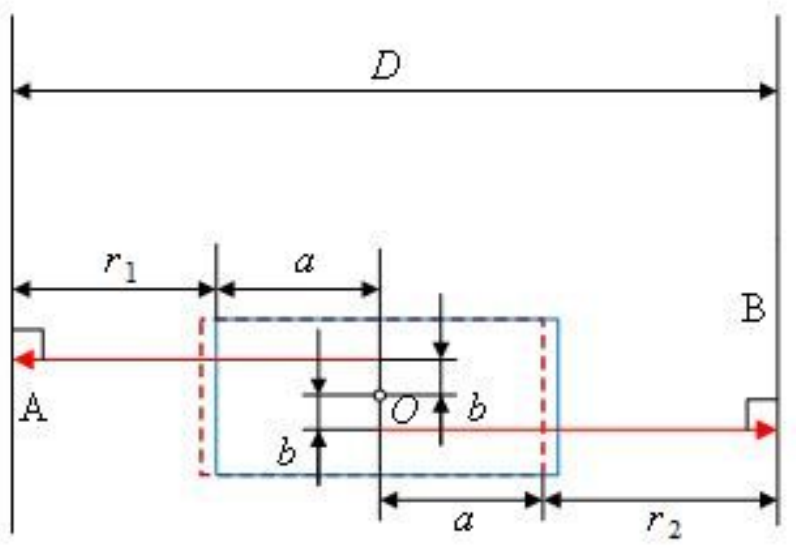

Figure 15

The calibration principle of parameter a.

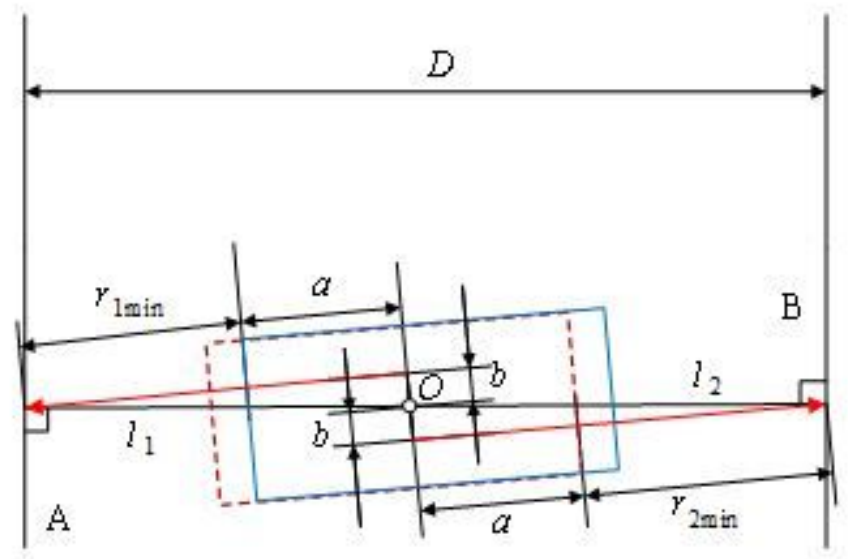

Figure 16

The calibration principle of parameter $b$. 


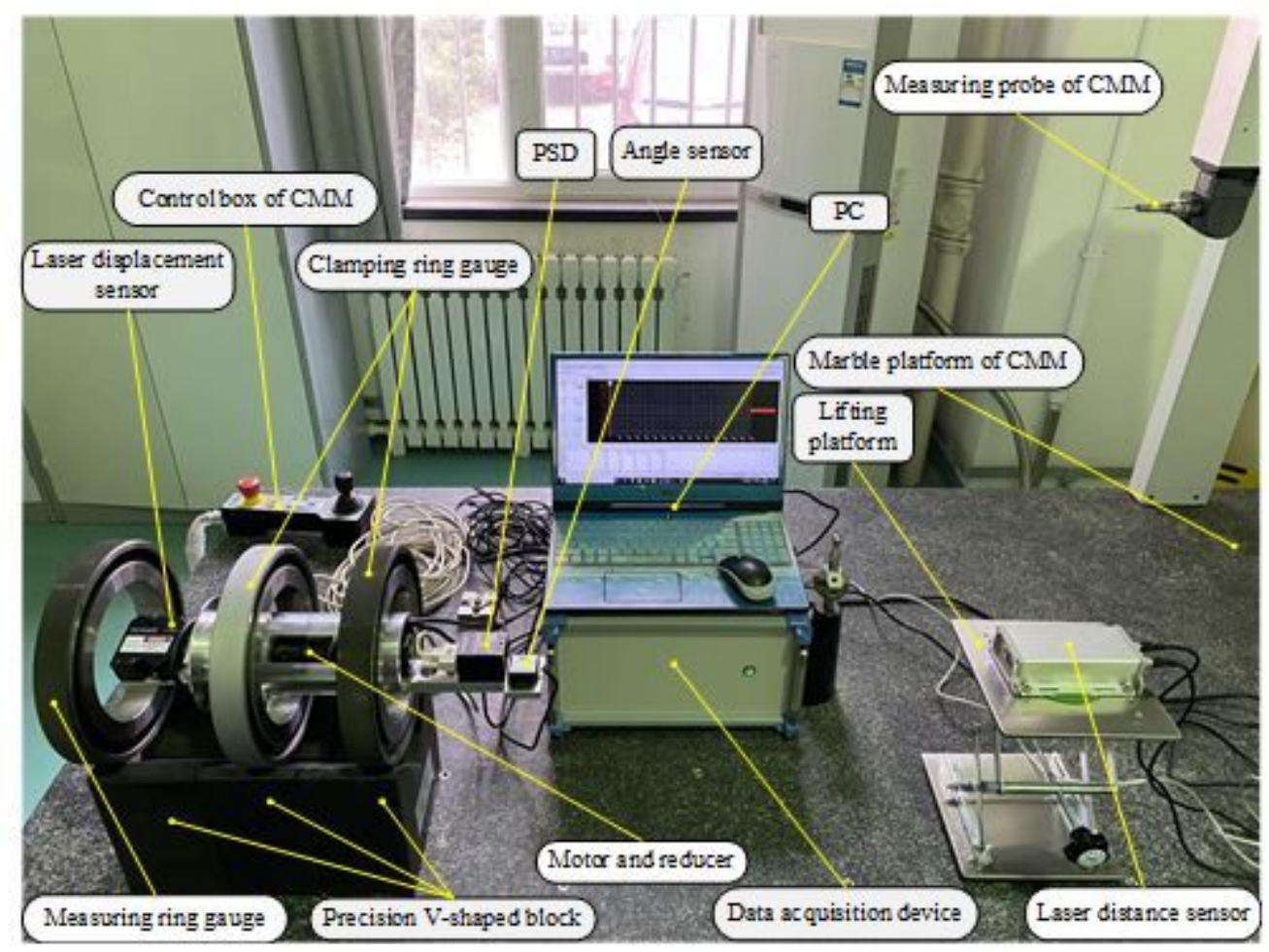

Figure 17

The measurement experimental scene with developed device. 

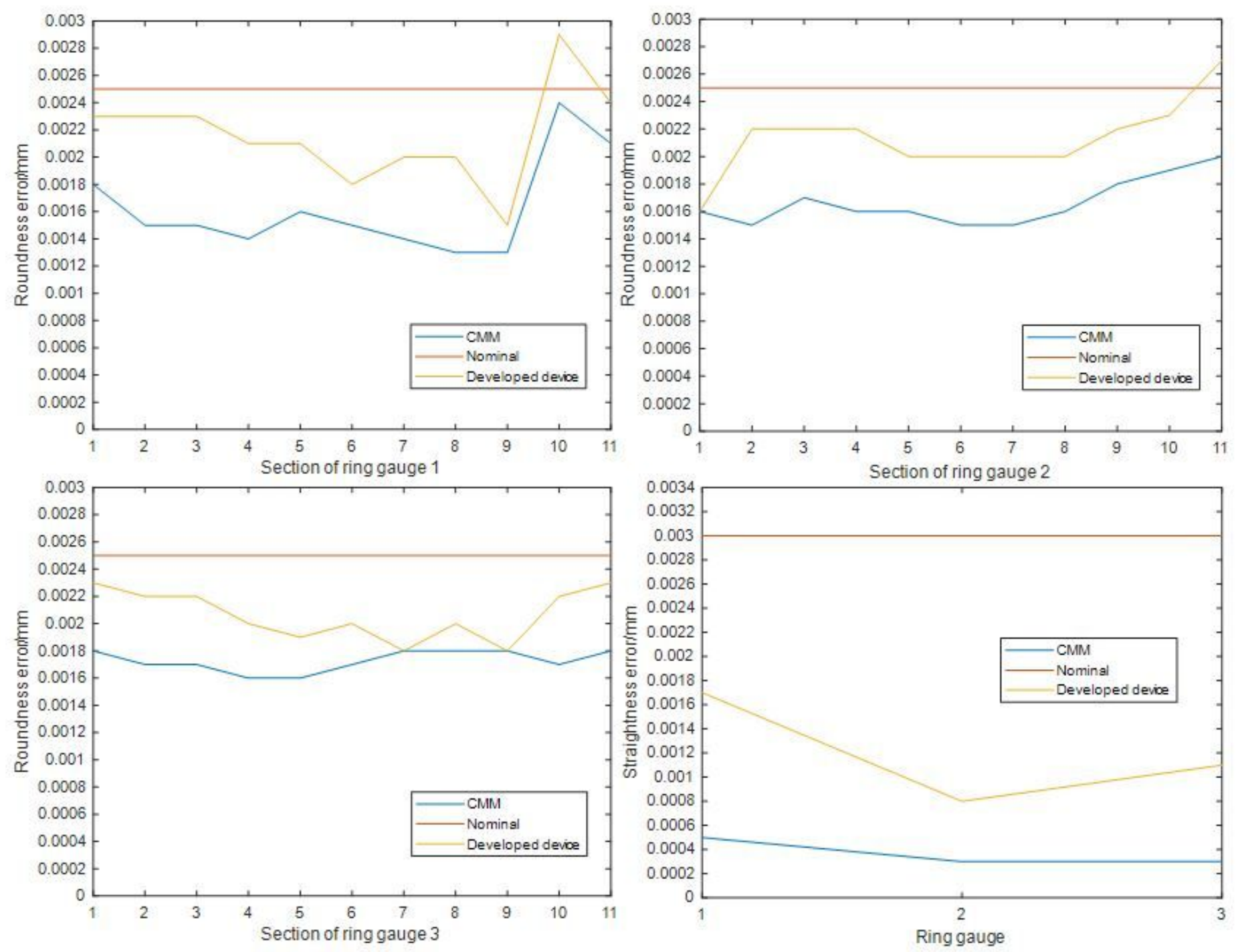

Figure 18

Comparison of measurement results. 